\title{
Functional significance of the discordance between transcriptional profile and left ventricular structure/function during reverse remodeling
}

\author{
Veli K. Topkara, ${ }^{1}$ Kari T. Chambers, ${ }^{1}$ Kai-Chien Yang, ${ }^{2}$ Huei-Ping Tzeng, ${ }^{1}$ Sarah Evans, ${ }^{1}$ \\ Carla Weinheimer, ${ }^{1}$ Attila Kovacs, ${ }^{1}$ Jeffrey Robbins, ${ }^{3}$ Philip Barger, ${ }^{1}$ and Douglas L. Mann ${ }^{1}$ \\ 'Center for Cardiovascular Research, Division of Cardiology, Department of Medicine, Washington University School of \\ Medicine, St. Louis, Missouri, USA. 'Department of Pharmacology, National Taiwan University School of Medicine, \\ Taipei, Taiwan. ${ }^{3}$ Molecular Cardiovascular Biology, Heart Institute, Cincinnati Children's Hospital, Cincinnati, Ohio, USA.
}

To elucidate the mechanisms for reverse LV remodeling, we generated a conditional (doxycycline [dox] off) transgenic mouse tetracycline transactivating factor-TRAF2 (tTA-TRAF2) that develops a dilated heart failure (HF) phenotype upon expression of a proinflammatory transgene, TNF receptor-associated factor 2 (TRAF2), and complete normalization of LV structure and function when the transgene is suppressed. tTA-TRAF2 mice developed a significant increase in LV dimension with decreased contractile function, which was completely normalized in the TTATRAF2 mice fed dox for 4 weeks (tTA-TRAF2 ${ }^{\text {dox4W }}$ ). Normalization of LV structure and function was accompanied by partial normalization ( $60 \%)$ of gene expression associated with incident HF. Similar findings were observed in patients with dilated cardiomyopathy who underwent reverse LV remodeling following mechanical circulatory support. Persistence of the HF gene program was associated with an exaggerated hypertrophic response and increased mortality in tTA-TRAF2 ${ }^{\text {dox4 }}$ mice following transaortic constriction (TAC). These effects were no longer observed following TAC in tTA-TRAF2 ${ }^{\mathrm{dox} 8 \mathrm{~W}}$, wherein there was a more complete $(88 \%)$ reversal of the incident HF genes. These results demonstrate that reverse $L V$ remodeling is associated with improvements in cardiac myocyte biology; however, the persistence of the abnormal HF gene program may be maladaptive following perturbations in hemodynamic loading conditions.

Authorship note: V.K. Topkara and K.T. Chambers contributed equally to this work.

Conflict of interest: The authors have declared that no conflict of interest exists.

Submitted: December 13, 2015 Accepted: March 7, 2016 Published: April 7, 2016

Reference information: JCI Insight. 2016;1(4):e86038. doi:10.1172/jii.insight.86038.

\section{Introduction}

As recently as 1980, it was widely held that no therapeutic intervention could reverse the dilated cardiomyopathic phenotype of the failing heart once it had developed, thus giving rise to the notion that the development of left ventricular (LV) remodeling and LV dysfunction were irreversible. However, more recent clinical studies have shown that medical and device therapies that reduce heart failure (HF) morbidity and mortality are accompanied by a reversal of the pathological LV remodeling (reverse LV remodeling; refs. 1, 2) that occurs during the development of HF. Reverse LV remodeling represents the summation of a series of integrated biological changes in cardiac myocyte size and function, as well as changes in LV structure and organization that allow for normalization of the LV end-diastolic pressure-volume (LVEDP-volume) relationship (reviewed in ref. 3). Although many of the individual components of reverse LV remodeling have been identified and annotated, prior studies have not clarified which of these cellular processes is required for the regression of pathological LV remodeling. Accordingly, to begin to dissect the biological mechanisms that are responsible for reverse LV remodeling, we developed and characterized a conditional transgenic mouse model tetracycline transactivating factor-TRAF2 (tTA-TRAF2) that develops a dilated HF phenotype when a proinflammatory transgene, TNF receptor-associated factor 2 (TRAF2), is constitutively expressed and that undergoes complete normalization of LV structure and function when the transgene is suppressed. Here, we show that complete normalization of LV structure and function is accompanied by salutary changes in the biology of the adult cardiac myocyte and that this structural and functional recovery is dissociated from changes in the extracellular matrix, as well as from reversal of gene 
ontogenies that become dysregulated during LV remodeling. We further show that the failing heart remains vulnerable to hemodynamic stress after restoration of LV structure and function and that this vulnerability diminishes over time, after normalization of the abnormal HF gene program.

\section{Results}

Characterization of $t T A-T R A F 2$ mice. Figure 1 shows that the tTA-TRAF2 mice develop a dilated HF phenotype at 12 weeks, characterized by an increased heart weight-to-body weight $(\mathrm{HW} / \mathrm{BW})$ ratio $(P<0.05)$, increased LV dilation $(P<0.05)$, decreased fractional shortening, and adverse LV remodeling as denoted by an increased radius-to-thickness $(\mathrm{r} / \mathrm{h})$ ratio, consistent with the phenotype observed with constitutive overexpression of TRAF2 using a Myh6 promoter (4). However, the salient finding shown by Figure 1 is that HW/BW, LV dilation, and depressed LV fractional shortening, as well as the $\mathrm{r} / \mathrm{h}$ ratio, were completely normalized in the tTA-TRAF2 ${ }^{\text {dox } 4 \mathrm{~W}}$ mice that were fed doxycycline (dox) for 4 weeks and were not statistically significant from littermate (LM) and $\mathrm{LM}^{\mathrm{dox} 4 \mathrm{~W}}$ control mice ( $P>0.05$ for all parameters). Light microscopic evaluation of the hearts from tTA-TRAF2 and tTA-TRAF2 ${ }^{\text {dox }}$ mice at 12 weeks revealed that the histological appearance was similar to LM and LM ${ }^{\text {dox4W }}$ controls. Notably, there was no discernible inflammatory infiltrate by H\&E staining (data not shown). Transmission electron microscopic evaluation of the 12-week-old LM and LM ${ }^{\text {dox4W }}$ hearts (Figure $1 \mathrm{H}$ ) revealed a characteristic linear array of sarcomeres and myofibrils. In contrast, the myofibrils in the 12-week-old tTA-TRAF2 mice were less organized with mild loss of sarcomere registration, disorganized mitochondrial cristae, and accumulation of protein aggregates - consistent with the ultrastructural changes previously reported in transgenic mice with TNF expression driven by Myh6 promotion (MHC-TNF mice) and transgenic mice with high copy TRAF2 expression driven by $M y h 6$ promotion (MHC-TRAF2 ${ }^{\mathrm{HC}}$ ) mice $(4,5)$. The transmission electron microscopic evaluation of the tTA-TRAF $2^{\mathrm{dox} 4 \mathrm{~W}}$ hearts revealed a linear arrangement of sarcomeres and myofibrils, disappearance of the protein aggregates, and improved organization of mitochondrial cristae.

Isolated cardiac myocyte and LV contractile function. Cardiac contractility was assessed at the level of the isolated cardiac myocyte and the intact LV chamber in 12-week-old mice by measuring sarcomere shortening in freshly isolated cardiac myocytes, as well as LV developed pressure (LVDP), $\mathrm{LV}+\mathrm{dP} / \mathrm{d} t$ and $\mathrm{LV}-\mathrm{dP} / \mathrm{d} t$ in isolated buffer-perfused Langendorff hearts. As shown by the representative sarcomere shortening curves in Figure 2A and group data shown in Figure 2B, there was a significant decrease $(P<0.05)$ in the extent of sarcomere shortening in myocytes from the tTA-TRAF2 mice when compared with myocytes from LM mice. Remarkably, the extent of sarcomere shortening in tTA-TRAF2 $2^{\text {dox } 4 \mathrm{~W}}$ myocytes was not significantly different from myocytes from $\mathrm{LM}^{\mathrm{dox} 4 \mathrm{~W}}$ control mice. Analysis of intact LV chamber function showed that the contractile function of the tTA-TRAF2 hearts was significantly depressed when compared with LM control mice. As shown in Figure 2, C and D, LVDP and LV $+\mathrm{dP} / \mathrm{d} t$ were significantly decreased $(P<0.05$ for both), and peak $-\mathrm{dP} / \mathrm{d} t$ was significantly greater $(P<0.05)$ in the tTA-TRAF2 mice compared with LM controls. Similar to our observations in isolated cardiac myocytes, $\mathrm{LVDP},+\mathrm{dP} / \mathrm{d} t$, and $-\mathrm{dP} / \mathrm{d} t$ returned to baseline values in the tTA-TRAF $2^{\mathrm{dox} 4 \mathrm{~W}}$ hearts and were not different statistically $(P>0.05$ for each parameter) when compared with $\mathrm{LM}^{\mathrm{dox} 4 \mathrm{~W}}$ controls. Analysis of LV pressure volume curves in Langendorff-perfused hearts showed that the LV pressure volume curves of the tTA-TRAF2 ${ }^{\mathrm{dox} 4 \mathrm{~W}}$ were shifted leftward when compared with tTA-TRAF2 mouse hearts (Supplemental Figure 1; supplemental material available online with this article; doi:10.1172/ jci.insight.86038DS1). Viewed together, these results suggest that there is a complete normalization of cardiac myocyte shortening, LV chamber contractility, and LV diastolic function in the TTA-TRAF2 ${ }^{\text {dox } 4 \mathrm{~W}}$ mice.

Extracellular matrix. Consistent with our prior observations in transgenic mice with constitutive expression of TRAF2, we observed a small but significant $(P<0.05)$ increase in fibrillar collagen content in the tTA-TRAF2 mice when compared with LM controls at 12 weeks of age (Figure 3). In contrast with the findings observed in terms of LV structure and function, the fibrillar collagen content did not regress in the tTA-TRAF2 ${ }^{\text {dox } 4 \mathrm{~W}}$ mouse hearts. As shown in Figure 3B, the fibrillar collagen content in the 12-week-old tTA-TRAF2 ${ }^{\mathrm{dox} 4 \mathrm{w}}$ mice was not significantly different $(P>0.05)$ from the tTA-TRAF2 and was significantly greater $(P<0.05)$ than 12 -week-old $\mathrm{LM}^{\mathrm{dox} 4 \mathrm{~W}}$ mice.

Gene expression profiling. To determine the changes in gene expression that were associated with reverse LV remodeling, we performed transcriptional profiling in 12-week-old LM, LM ${ }^{\text {dox4 }}$, tTA-TRAF2, and tTA-TRAF2 ${ }^{\text {dox }} 4 \mathrm{~W}$ mouse hearts. Because of the effects of dox on gene expression (6), we compared changes in gene expression in the tTA-TRAF2 mice vs. LM control mice to characterize genes that were associated with LV remodeling, and we compared changes in gene expression in tTA-TRAF2 ${ }^{\text {dox } 4 \mathrm{~W}}$ vs. $\mathrm{LM}^{\text {dox } 4 \mathrm{~W}}$ mice to 
A

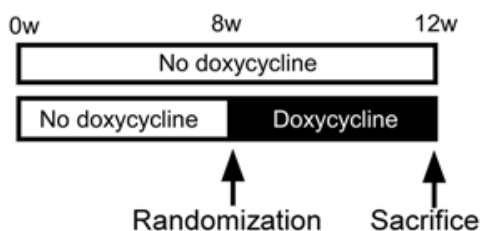

LM

TTA-TRAF2

E

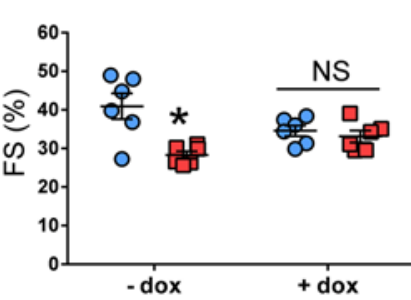

H
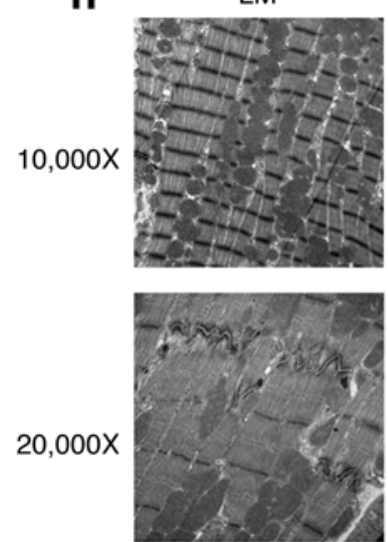

B

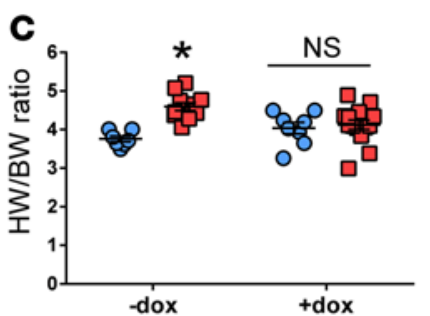

$\mathbf{F}$

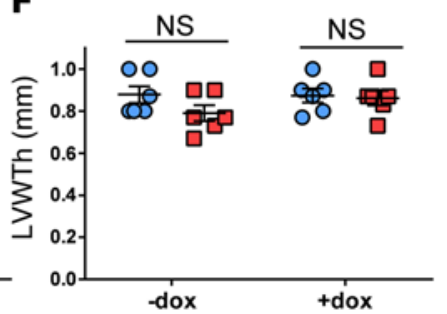

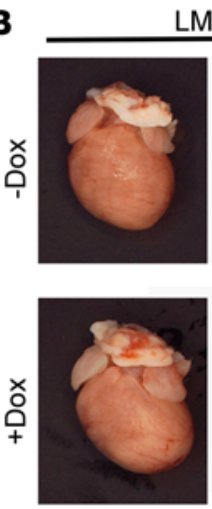

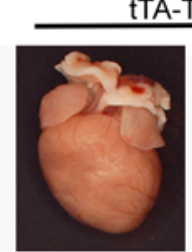

tTA-TRAF2
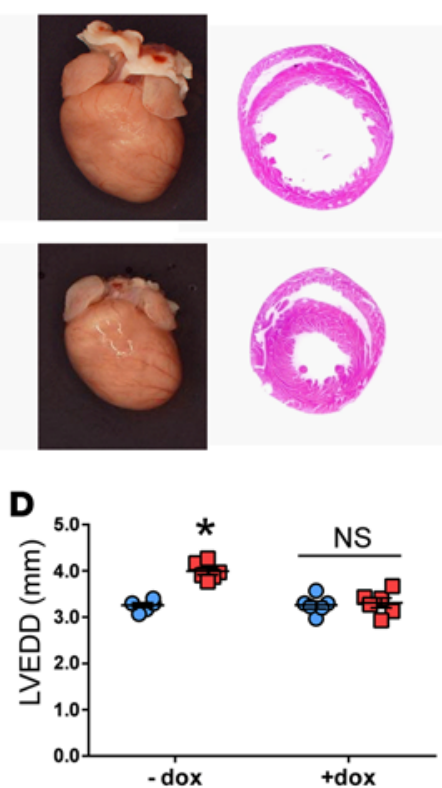

G

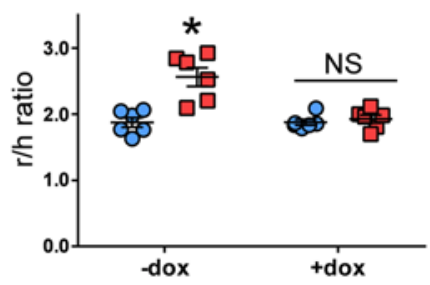

TTA-TRAF2 ${ }^{\text {dox }}$
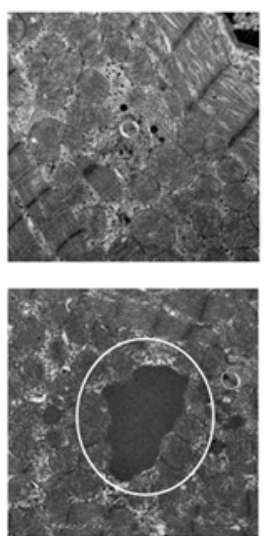
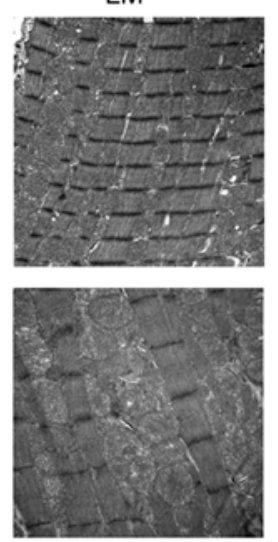
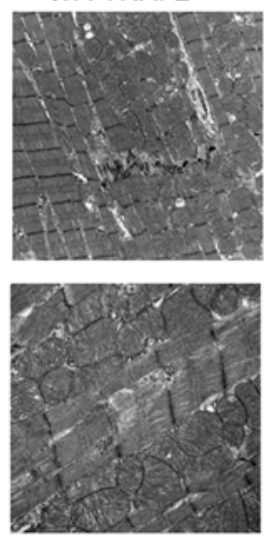

Figure 1. Characterization of tTA-TRAF2 mice. (A) tTA-TRAF2 mice and LM controls were followed up to 8 weeks of age and were then randomized to receive doxycycline in the drinking water or no doxycycline for 4 weeks. (B) Photographs of whole hearts and sagittal sections of LM, tTA-TRAF2, LM ${ }^{\text {dox4W }}$, and tTA-TRAF2 ${ }^{\text {dox4W }}$ mouse hearts at 12 weeks of age. (C) Heart weight-to-body weight ratio (HW/BW). (D) Left ventricular end-diastolic diameter (LVEDD).(E) LV fractional shortening percent (FS\%). (F) LV wall thickness (LVWTh). (C) r/h ratio of LM, tTA-TRAF2, LM ${ }^{\text {dox4W }}$, and tTA-TRAF2 ${ }^{\text {dox4W }}$ at 12 weeks of age ( $n=6$ mice/group). (H) Representative transmission electron micrographs from 12-week LM, tTA-TRAF2, $\mathrm{LM}^{\mathrm{dox} 4 \mathrm{~W}}$, and TTA-TRAF2 $2^{\mathrm{dox} 4 \mathrm{~W}}$ mice at $\times 10,000$ and $\times 20,000$ magnification. Protein aggregates are enclosed by the circle. Statistical analysis was performed using 2-way ANOVA with Tukey post-hoc analysis. ( ${ }^{*} P \leq 0.05$ compared with LM control.) LM, littermate; LM ${ }^{\text {dox4W }}$, LM mouse fed doxycycline for 4 weeks; $r / h$ ratio, radius/wall thickness. 
A

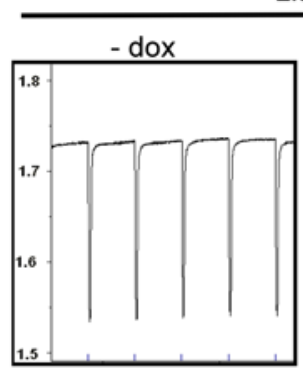

LM

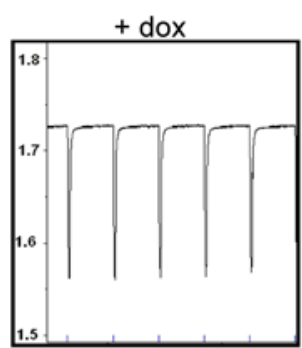

TTA-TRAF2
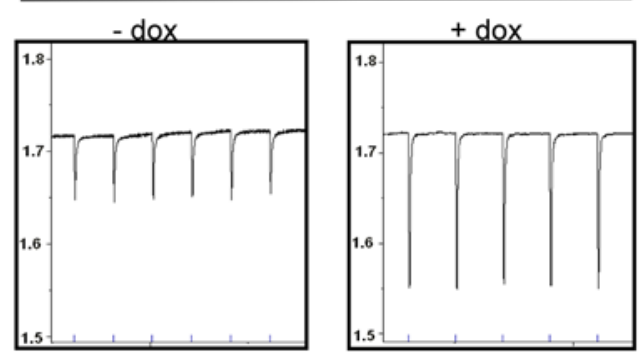

B

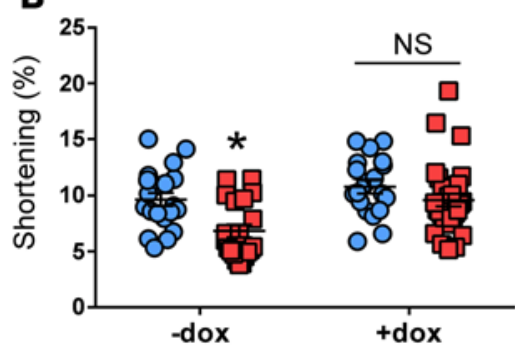

C
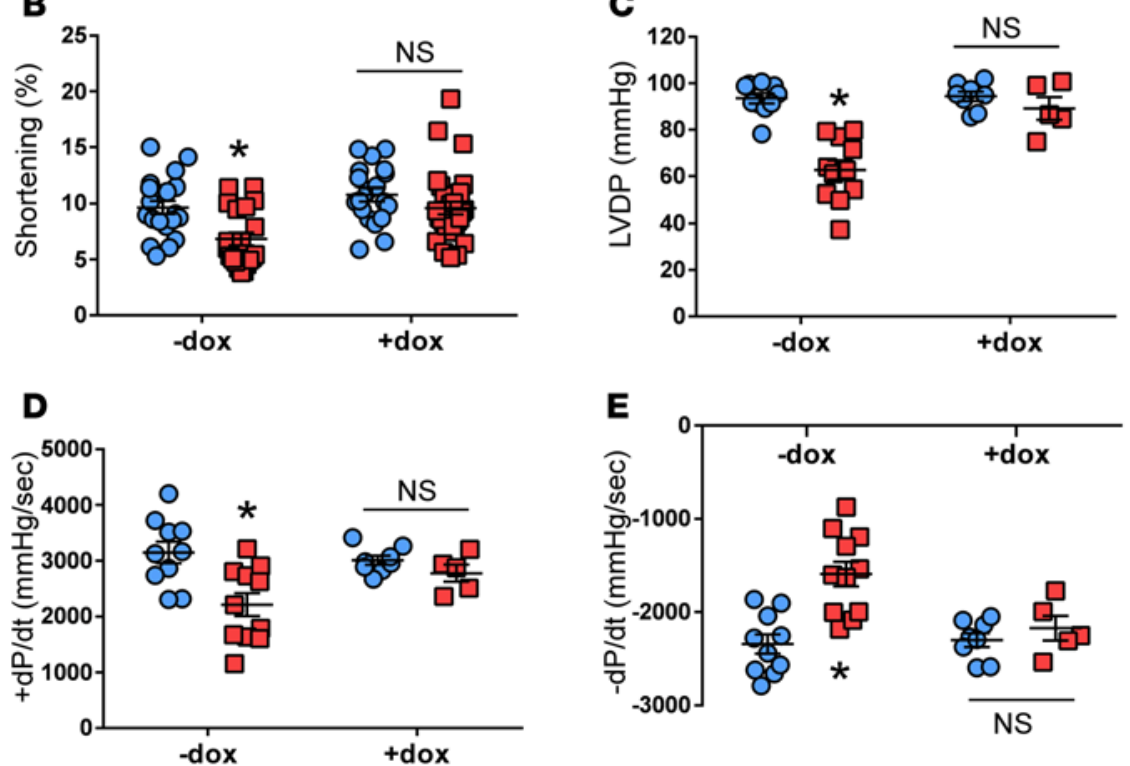

Figure 2. Myocyte and LV chamber contractile function of tTA-TRAF2 mice. (A) Representative tracings of sarcomere shortening in isolated cardiac myocytes and (B) group data of sarcomere shortening from LM, tTA-TRAF2, LM ${ }^{\text {dox4W }}$, and tTA-TRAF2 ${ }^{\mathrm{dox} 4 \mathrm{~W}}$ mouse hearts at 12 weeks of age (5-6/cells/heart from 3 hearts/group). (C) Left ventricular developed pressure (LVDP). (D) Peak $+\mathrm{dP} / \mathrm{d} t$ and (E) peak -dP/dt in 12-week LM $(n=10), \operatorname{tTA}-T R A F 2(n=8), \mathrm{LM}^{\mathrm{dox} 4 \mathrm{~W}}(n=11)$, and tTA-TRAF2 $^{\text {dox4W }}(n=5)$ mouse hearts. Statistical analysis performed using 2-way ANOVA with Tukey post-hoc analysis. $\left({ }^{*} P<0.05\right.$ compared with LM controls.)

characterize changes in genes that were associated with reverse LV remodeling. The salient finding shown by Figure $4 \mathrm{~A}$ is that there were 3,647 genes with significantly altered expression in the LV remodeled hearts $(P<0.05)$, referred to here as incident HF genes, whereas there were 3,383 genes with significantly altered expression in the reverse LV remodeled hearts. $(P<0.05)$. Of the 3,647 incident HF genes, $58 \%$ $(2,109 / 3,647)$ normalized in the tTA-TRAF $2^{\text {dox }}$ mice, and hence represent a subset of reversed incident HF genes. In contrast, $42 \%(1,538 / 3,647)$ of the incident HF genes were also expressed in tTA-TRAF2 $2^{\mathrm{dox} 4 \mathrm{~W}}$ mice, and thus represent a subset of incident HF genes that did not normalize during reverse LV remodeling, which we refer to here as persistently dysregulated HF genes. Interestingly, 55\% $(1,845 / 3,383)$ of the genes differentially regulated in the reverse LV remodeled hearts were not detectable during LV remodeling (i.e., incident $\mathrm{HF}$ genes) and thus represent a set of genes that are unique to reverse $\mathrm{LV}$ remodeling (see online Supplemental Table 1 for further characterization).

Unsupervised hierarchical clustering of expression levels of changes in gene expression in the LM, tTA-TRAF2, LM ${ }^{\mathrm{dox} 4 \mathrm{w}}$, and TRAF2 ${ }^{\mathrm{dox} 4 \mathrm{w}}$ mice showed that the gene expression profile of the tTA-TRAF$2^{\mathrm{dox} 4 \mathrm{~W}}$ hearts were distinct from the gene profiles of the LM ${ }^{\mathrm{dox} 4 \mathrm{~W}}$ hearts and the tTA-TRAF2 hearts (Figure 4B). Importantly, the gene expression profile of the tTA-TRAF2 $2^{\mathrm{dox} 4 \mathrm{~W}}$ mice clustered with the tTA-TRAF2 mice, consistent with incomplete reversal of the incident HF gene profile in the hearts that underwent reverse LV remodeling. Also, unsupervised hierarchical clustering of the gene profiles for the LM and $\mathrm{LM}^{\mathrm{dox}}{ }^{\mathrm{dW}}$ clustered together, suggesting that dox had no dramatic effect on myocardial gene expression at 
A

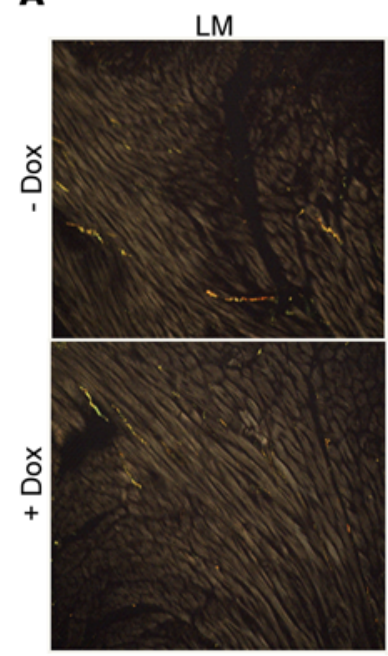

TTA-TRAF2

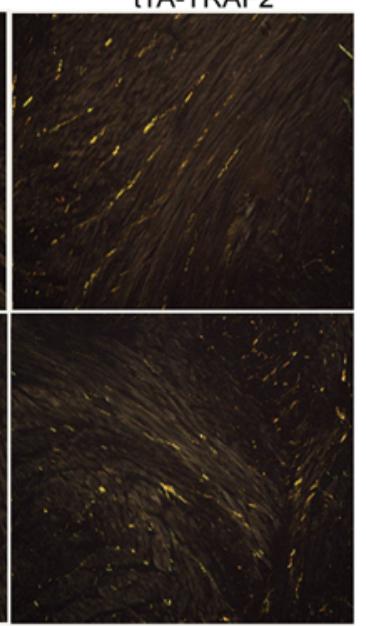

B $\square$ LM

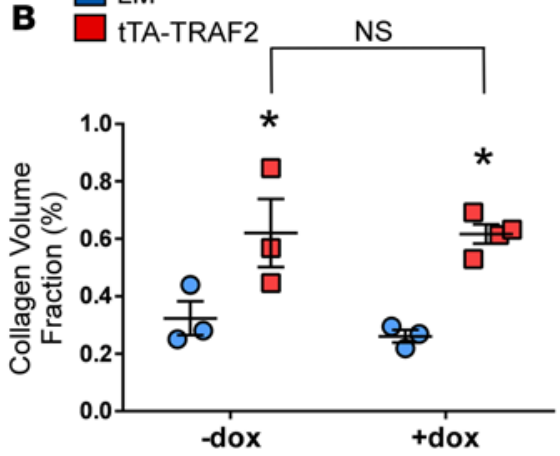

Figure 3. Myocardial fibrillar collagen content in tTA-TRAF2 mice. (A) Representative picrosirius red staining $(\times 400)$ for myocardial fibrillar collagen content and (B) group data for $\%$ collagen volume for LM, tTATRAF2, LM ${ }^{\text {dox } 4 \mathrm{~W}}$, and tTA-TRAF$2^{\text {dox4W }}$ mouse hearts ( $9-10$ fields analyzed for $n=3$ mice/group). Statistical analysis performed using 2-way ANOVA with Tukey post-hoc analysis. ( ${ }^{*} P<0.05$ vs. LM control.)

baseline. To further explore the differences in gene profiles in the LV remodeled and reverse LV remodeled cardiac phenotypes, we performed a principal component analysis (PCA). As shown in Figure 4C, the values for the PCA vectors for the $\mathrm{LM}$ and $\mathrm{LM}^{\text {dox4 }}{ }^{\mathrm{W}}$ hearts grouped closely together and were aligned differently from the values for the PCA vectors for the tTA-TRAF2 and tTA-TRAF2 ${ }^{\text {dox } 4 \mathrm{w}}$ hearts. Consistent with the findings with hierarchical clustering, the PCA profiles for the tTA-TRAF2 and the tTA-TRAF2 ${ }^{\mathrm{dox} 4 \mathrm{~W}}$ hearts were overlapping.

Kyoto Encyclopedia of Genes and Genomes (KEGG) functional analysis identified significant changes in incident HF gene expression in pathways implicated in both hypertrophic $\left(P=3.3 \times 10^{-2}\right)$ and dilated cardiomyopathy $(\mathrm{DCM})\left(P=2.7 \times 10^{-2}\right)$ in the tTA-TRAF2 $2^{\mathrm{dox} 4 \mathrm{~W}}$ reverse LV remodeled hearts (Supplemental Figure 2). Figure 4D displays a diagram of the cardiac hypertrophy/DCM-related cardiac myocyte genes displayed as either reversed incident HF genes or persistently dysregulated incident HF genes (See Supplemental Table 2 for gene lists), whereas Figure 4E illustrates a heat map of the changes in the reversed and persistently dysregulated incident HF genes, grouped functionally into 1 of 5 different cardiac myocyte gene modules, including extracellular matrix, integrin/cytoskeleton, sarcomere, excitation-contraction coupling, and metabolism. Evaluation of the data in Figure 4, D and E, permits several interesting observations, the first of which is that normalization of cardiac myocyte shortening, LV chamber pump performance, and LV structure during reverse LV remodeling is not associated with a single gene or a single gene module. Rather, reversal of the LV HF phenotype is accompanied by significant changes in expression in multiple genes residing with each of the gene modules that were examined, save for genes involved in metabolism. Second, the changes in reversed incident HF genes were greatest for genes in the extracellular matrix/collagen module (18\%), followed by the modules for integrin/cytoskeleton (13\%), sarcomere (7\%), excitation-contraction coupling (6\%), and metabolism (0\%). Conversely, the gene modules that had the greatest percentage of persistently dysregulated incident HF genes were related to metabolism (27\%), followed by sarcomere (20\%), integrin/cytoskeleton (13\%), matrix collagen (10\%), and excitation contraction coupling (5\%).

Response to pressure overload. To determine the functional significance of the abnormal gene expression profile in the tTA-TRAF2 $2^{\text {dox } 4 \mathrm{~W}}$ mice, we subjected 12 -week-old tTA-TRAF2 ${ }^{\mathrm{dox} 4 \mathrm{~W}}$ and $\mathrm{LM}^{\mathrm{dox} 4 \mathrm{~W}}$ mice to transaortic constriction (TAC). The important finding shown in Figure 5 is that superimposition of a pressure overload resulted in an exaggerated hypertrophic phenotype in the tTA-TRAF2 ${ }^{\mathrm{dox}} 4 \mathrm{~W}$ mice, marked by a significant increase in LV mass index $(P<0.03)$ and LV wall thickness $(P<0.02) 4$ weeks following TAC. In contrast, there were no significant differences in LV end-diastolic dimension (LVEDD) and the $\mathrm{r} / \mathrm{h}$ ratio in the tTA-TRAF2 $2^{\mathrm{dox} 4 \mathrm{~W}}$ and $\mathrm{LM}^{\mathrm{dox} 4 \mathrm{~W}}$ mice 4 weeks after TAC. Importantly, the exaggerated hypertrophic response in the pressure overloaded tTA-TRAF2 $2^{\mathrm{dox} 4 \mathrm{~W}}$ mice was associated with a significant increase in mortality in the tTA-TRAF2 ${ }^{\text {dox } 4 \mathrm{~W}}$ mice when compared with $\mathrm{LM}^{\mathrm{dox} 4 \mathrm{~W}}$ mice $(P<0.04)$, shown by the Kaplan-Meier analysis in Figure 5E. 
A

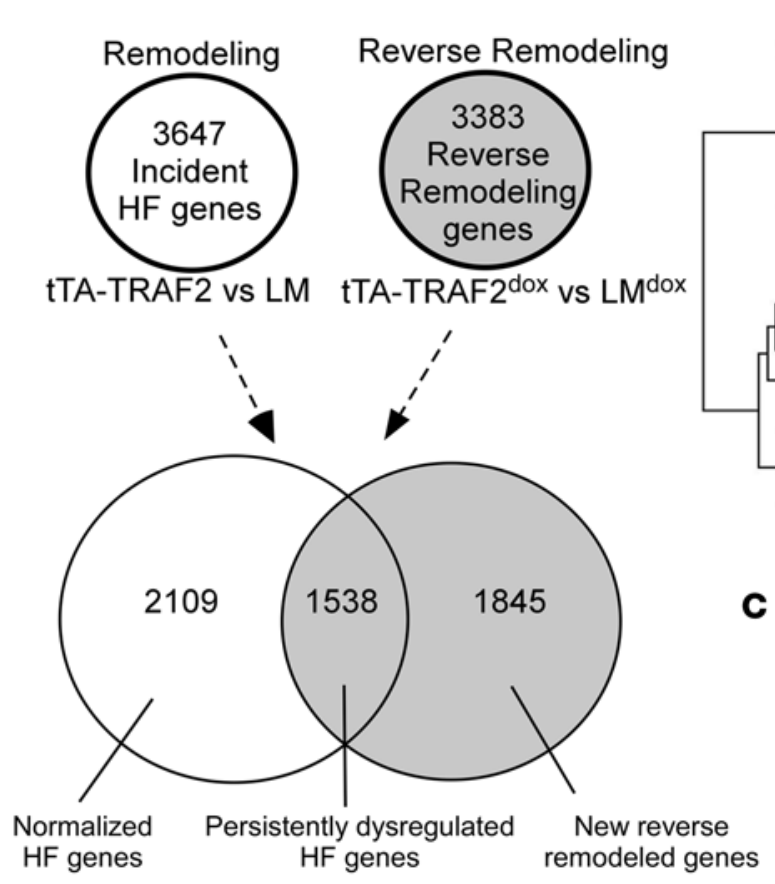

B
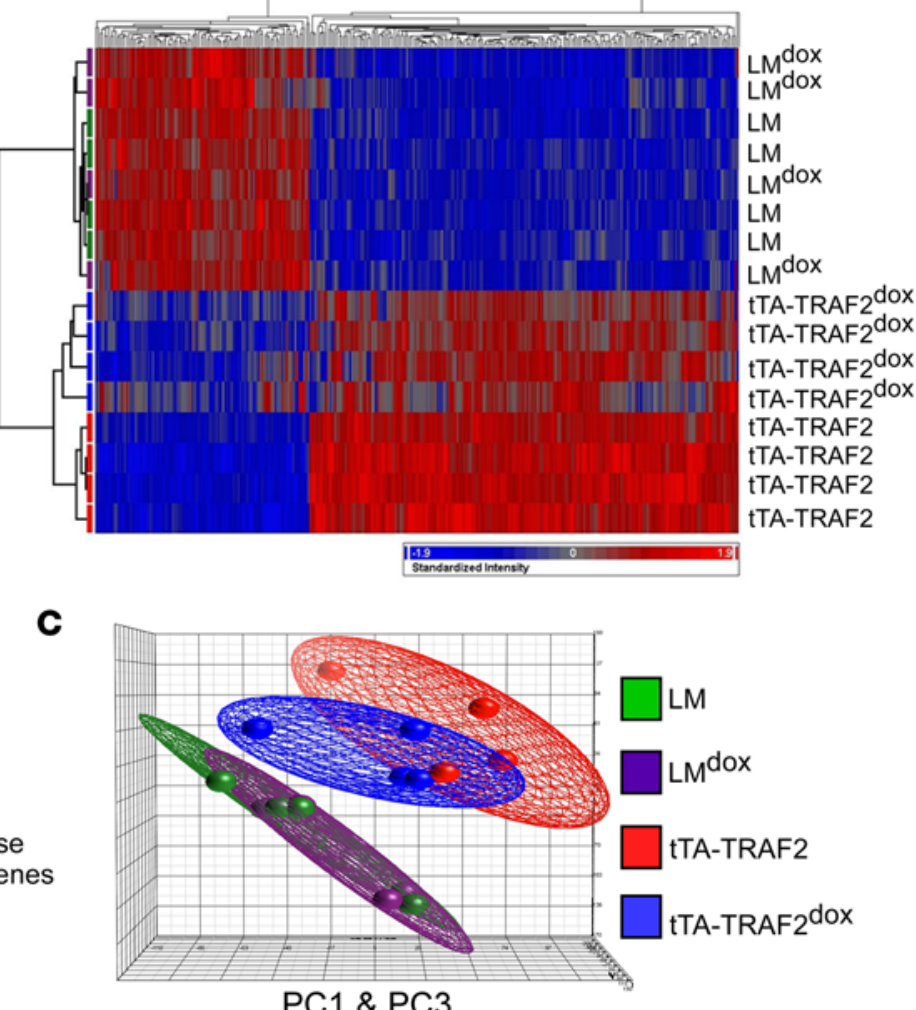

$\mathbf{E}$

Matrix/Collagen (40) Integrin/Cytoskeleton (32)
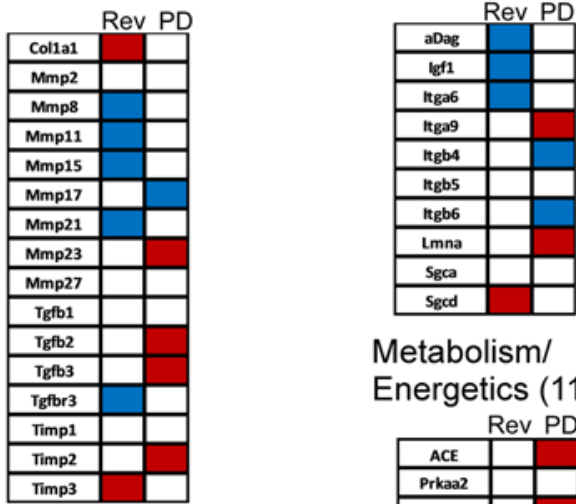

Metabolism/

Energetics (11)

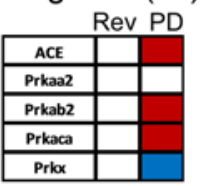

Coupling (35)

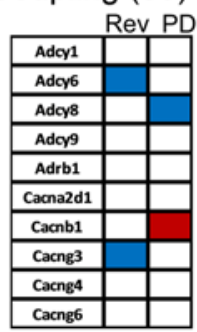

Sarcomere (15)

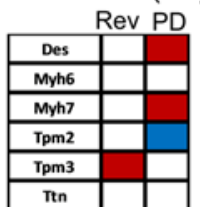

Increased expression

Decreased expression genes in relation to their anatomic location within the cardiac myocyte. (E) Illustration of persistently dysregulated and reversed HF genes in gene modules relating to cardiac myocyte function (see text for details). The number of genes within each module is given in parenthesis ( $n=4$ hearts per group). Red indicates increased gene expression; blue indicates decreased gene expression relative to $\mathrm{LM}$; Rev, reversed HF genes; $\mathrm{PD}$, persistently dysregulated HF genes. Analysis performed with Partek Genomics Suite. LM, littermate; $\mathrm{LM}^{\mathrm{dox} 4 \mathrm{~W}}$, LM mouse fed doxycycline for 4 weeks; $\mathrm{r} / \mathrm{h}$ ratio, radius/wall thickness, PC, principal component; PD, persistently dysregulated; Rev, reversed. 

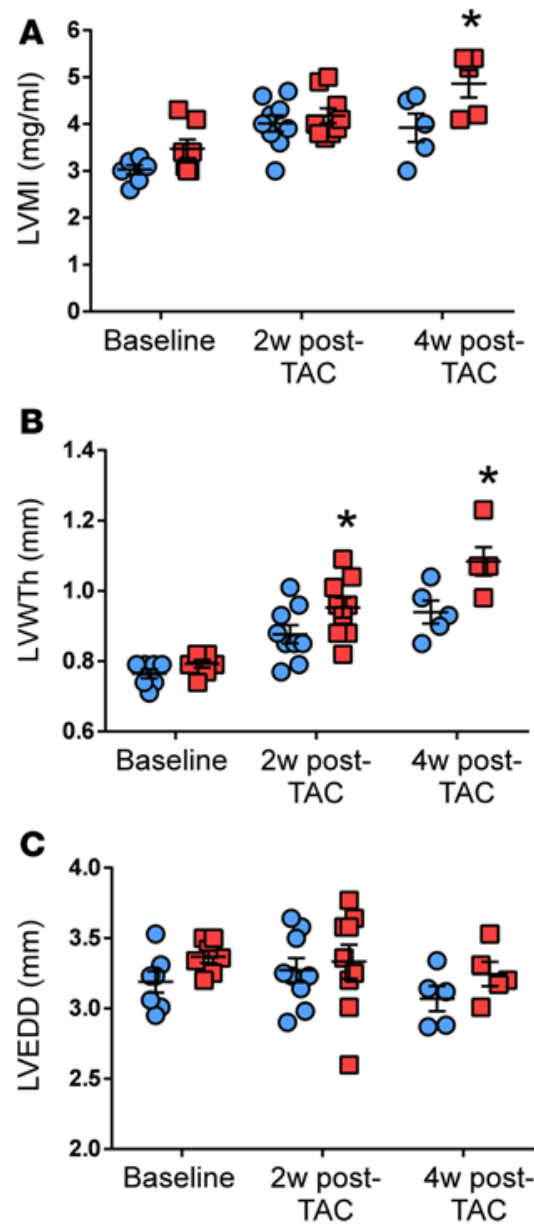

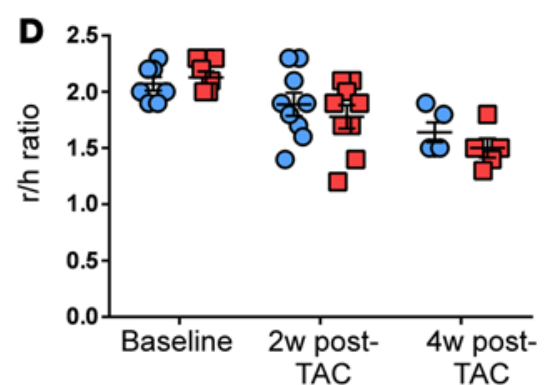

E

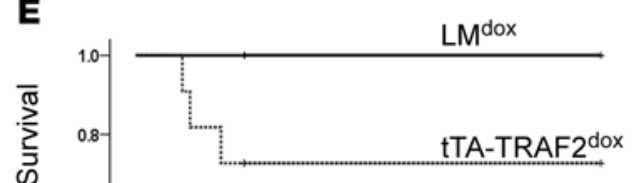

Log-rank $p=0.04$
Figure 5. Effect of transaortic constriction (TAC) on LV structure and mortality in LM ${ }^{\text {dox4 }} \mathrm{W}$ and tTA-TRAF2 ${ }^{\text {dox4w }}$ mice. (A) LV mass index (LVMI), (B) LV wall thickness (LVWTh), (C) LV end-diastolic diameter (LVEDD), and (D) LV r/h ratio at 2 and 4 weeks after TAC (baseline $n=7,2$ weeks after TAC $n$ $=8-9,4$ weeks after TAC $n=5)$. (E) Kaplan-Meier analysis of survival in LM $^{\text {dox }}$ and tTA-TRAF2 ${ }^{\text {dox }}$ mice following TAC ( $n=12$ LM $^{\text {dox4W }}$ hearts and $n=14$ tTA-TRAF2 ${ }^{\text {dox } 4 \mathrm{~W}}$ hearts). Statistical analysis performed using a repeated measures analysis using mixed models methodology (See Methods section). ( ${ }^{*} P<0.05$ )

Time-dependent changes in gene expression in reverse $L V$ remodeling. To investigate whether the expression of dysregulated HF genes in the reverse LV remodeled tTA-TRAF2 ${ }^{\text {dox } 4 \mathrm{~W}}$ mouse hearts was time dependent, we administered dox for an additional 4 weeks (a total of 8 weeks of treatment) in a second cohort of mice and then repeated transcriptional profiling. Similar to our findings in the 12-week-old tTA-TRAF2 mice, there were 3,095 incident HF genes in the 16-week-old tTA-TRAF2 ${ }^{\text {dox } 8 \mathrm{~W}}$ mouse hearts. However, of the 3,095 incident HF genes, $88 \%(2,724 / 3,095)$ of these genes normalized in the tTA-TRAF2 ${ }^{\text {dox } 8 \mathrm{~W}}$ mice, whereas only $12 \%(371 / 3,095)$ of the incident HF genes remained persistently dysregulated HF in the tTA-TRAF2 ${ }^{\text {dox } 8 \mathrm{~W}}$ mouse hearts (Figure 6). Interestingly, $70 \%(885 / 1,256)$ of the differentially expressed genes in the reverse LV remodeled hearts were not detected during LV remodeling and thus represent a set of genes that were unique to reverse LV remodeling genes (see online Supplemental Table 3 for further characterization).

In contrast to the findings observed following 4 weeks of dox treatment, unsupervised hierarchical clustering showed that the gene profiles for the LM, LM ${ }^{\text {dox } 8 \mathrm{~W}}$, and $\mathrm{tTA}-\mathrm{TRAF} 2^{\mathrm{dox} 8 \mathrm{~W}}$ hearts grouped together, whereas the genes for the tTA-TRAF2 mice clustered separately, suggesting that longer administration of dox led to more complete normalization of gene expression in the tTA-TRAF2 ${ }^{\text {dox8 }}{ }^{\text {mice }}$ (Figure 6C). To further explore the differences in gene profiles in the LV remodeled and reverse LV remodeled cardiac phenotypes, we performed a PCA. As shown in Figure 6D, the values for the PCA vectors for the $\mathrm{LM}, \mathrm{LM}^{\mathrm{dox} 8 \mathrm{w}}$, and tTA-TRAF2 ${ }^{\mathrm{dox} 8 \mathrm{~W}}$ hearts clustered together and were separate from the values for the PCA vectors for the tTA-TRAF2 hearts, consistent with the findings obtained with hierarchical clustering shown in Figure 6B. Interestingly, a KEGG functional analysis of the changes in gene expression (Supplemental Figure 3) again identified significant changes in gene expression pathways implicated in both hypertrophic $\left(P=6.3 \times 10^{-2}\right)$ and DCM $\left(P=1.1 \times 10^{-2}\right)$ in the reversed incident HF genes in the tTA-TRAF2 ${ }^{\text {dox }}$ hearts. Figure $6 \mathrm{E}$ illustrates a diagram of cardiac hypertrophy/ DCM-related cardiac myocyte genes displayed as reversed incident HF genes and persistently dysregulated HF genes in the reverse LV remodeled tTA-TRAF2 ${ }^{\text {dox } 8 \mathrm{~W}}$ mouse hearts (See Supplemental Table 
4 for gene lists). Figure $6 \mathrm{~F}$ shows a heat map of changes in the reversed and persistently dysregulated genes grouped into 1 of 5 different functionally related cardiac myocyte gene modules. Figure 7A compares and contrasts the changes in the number of reversed and persistently dysregulated genes in the tTA-TRAF2 $2^{\text {dox }}$ hearts following 4 and 8 weeks of dox. The important finding revealed by panel 7A is that there was an approximately 2 -fold increase in the number of reversed incident HF genes and an approximately 5 -fold decrease in the dysregulated HF genes in the tTA-TRAF2 ${ }^{\text {dox } 8 \mathrm{~W}}$ hearts when compared with tTA-TRAF ${ }^{\text {dox } 4 \mathrm{~W}}$ hearts. Figure $7 \mathrm{~B}$ summarizes the percentage of genes in each module that normalized after 4 and 8 weeks of dox. As show, there was a greater reversal in incident HF genes for each of the gene modules after 8 weeks of treatment, with the greatest percentage of reversed incident $\mathrm{HF}$ genes for the sarcomeric genes $(\sim 35 \%)$. Figure $7 \mathrm{C}$ shows that there was a striking decrease in the percentage of persistently dysregulated HF genes within each of the 5 gene modules following 8 weeks of treatment with dox. Indeed, save for the persistently dysregulated incident HF metabolism genes, all of the other persistently dysregulated genes in the cardiac myocyte gene modules were $<3 \%$ at 8 weeks. Of note, while $12 \%$ of the overall genes remained persistently dysregulated in the tTA-TRAF $2^{\mathrm{dox} 8 \mathrm{~W}}$ mice treated with dox for 8 weeks, $<1 \%$ of these genes were related to LV structure and/or function.

To determine the functional significance of the abnormal gene expression profile in the tTA-TRAF2 ${ }^{\mathrm{dox} 8 \mathrm{~W}}$ mice, we performed TAC on tTA-TRAF2 ${ }^{\mathrm{dox} 8 \mathrm{~W}}$ and $\mathrm{LM}^{\mathrm{dox} 8 \mathrm{~W}}$ mice. Figure 8 shows that superimposition of a hemodynamic pressure overload resulted in a significant overall increase in LV mass index $(P<0.001)$ and LV wall thickness $(P<0.001)$, along with a significant decrease in the $\mathrm{r} / \mathrm{h}$ ratio $(P=0.005)$ in the $\mathrm{LM}^{\mathrm{dox} 8 \mathrm{~W}}$ and tTA-TRAF2 ${ }^{\mathrm{dox} 8 \mathrm{w}}$ mouse hearts. Importantly, however, there was no significant difference in the LV mass index $(P=0.12)$, LV wall thickness $(P=0.07)$, LVEDD $(P=0.86)$, and $\mathrm{r} / \mathrm{h}$ ratio $(P=0.28)$ between the tTA-TRAF2 $2^{\text {dox8w }}$ and $\mathrm{LM}^{\text {dox8w }}$ mouse hearts. Kaplan-Meier analysis showed that there was also not a significant difference in mortality in the tTA-TRAF $2^{\text {dox } 8 \mathrm{~W}}$ mice when compared with $\mathrm{LM}^{\text {dox8 }}{ }^{\mathrm{W}}$ mice $(P=0.54)$. Thus, a greater degree of normalization of the persistently dysregulated genes in the tTA-TRAF $2^{\text {dox } 8 \mathrm{~W}}$ mouse hearts was associated with a normalization of the physiological response to a superimposed hemodynamic pressure overload.

To determine whether the observed changes in gene expression during reverse remodeling in the tTATRAF $2^{\text {dox }}$ hearts were also observed in human HF, we examined changes in gene expression in the LV of patients with DCM before and after hemodynamic unloading with a left ventricular assist device (LVAD), in comparison to nonfailing (NF) human LV that could not be transplanted for technical reasons. We have reportedly previously that the LVAD-supported hearts used for these studies undergo reverse LV remodeling following LVAD support; however, none of these patients could be weaned from LVAD support prior to cardiac transplantation (7). Figure 9A shows there were 1,928 incident HF genes in the failing human hearts and that $42 \%(810 / 1,928)$ of these genes normalized following LVAD support, whereas $58 \%$ $(1,118 / 1,928)$ of the incident HF genes remained persistently dysregulated. Analogous to changes in gene expression observed in reverse LV remodeling in the tTA-TRAF2 ${ }^{\text {dox }}$ mice treated with dox for 4 weeks, $60 \%(1,670 / 2,788)$ of the differentially expressed genes in the LVAD-supported hearts were new reverse remodeling genes (see Supplemental Table 5 on online supplement). Unsupervised hierarchical clustering (Figure 9B) of mRNA expression in the NF, DCM, and DCM after LVAD showed that the gene expression profiles of the LVAD-supported DCM hearts were indistinguishable from that of the pre-LVAD supported hearts. Similarly, the PCA analysis showed that the values for the PCA vectors of the DCM and post-LVAD DCM hearts were grouped together and were distinct from the PCA vectors for NF human hearts. Figure 9D shows that 23 of 25 (92\%) cardiac hypertrophy/DCM incident HF genes were persistently dysregulated in the DCM-LVAD supported hearts, whereas only 2 of $25(8 \%)$ of the incident HF myocyte genes were reversed. The only gene module with reversal of incident HF genes was the sarcomere gene module, with 2 of $15(13 \%)$ of the incident HF genes reversed (Figure 9E). Of note, the greatest overlap of dysregulated human HF genes with the dysregulated genes in the tTA-TRA2 ${ }^{\text {dox } 4 \mathrm{w}} / \mathrm{tTA}^{\mathrm{T}} \mathrm{TRA} 2^{\mathrm{dox} 8 \mathrm{~W}}$ hearts for the metabolism/energetics gene module ( $100 \%$ human genes detected in the mouse models), followed by the sarcomere gene module ( $75 \%$ human genes detected in the mouse models), and lesser overlap for the gene modules for matrix/cytoskeleton ( $43 \%$ human genes detected in the mouse models), excitation contraction coupling ( $33 \%$ human genes detected in the mouse models), and integrin/cytoskeleton ( $25 \%$ human genes detected in the mouse models). However, this comparison must be regarded as provisional because of the different platforms used for transcriptional profiling, as well as the differences in tissue handling of the murine and human myocardial samples. 
A

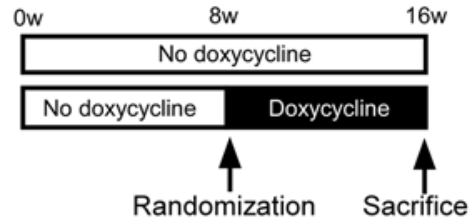

B Remodeling

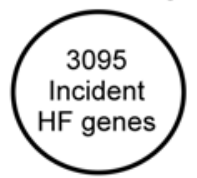

tTA-TRAF2 vs LM

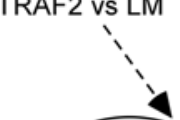

,

Reverse Remodeling

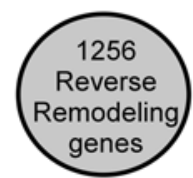

TA-TRAF2 ${ }^{\text {dox }}$ vs LM ${ }^{\text {dox }}$

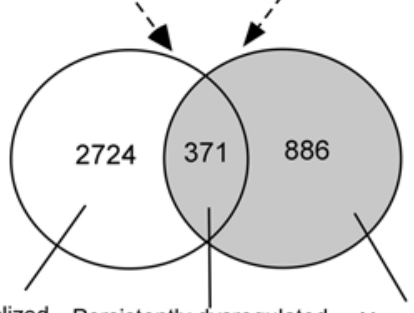

Normalized Persistently dysregulated New reverse HF genes HF genes remodeled genes

E

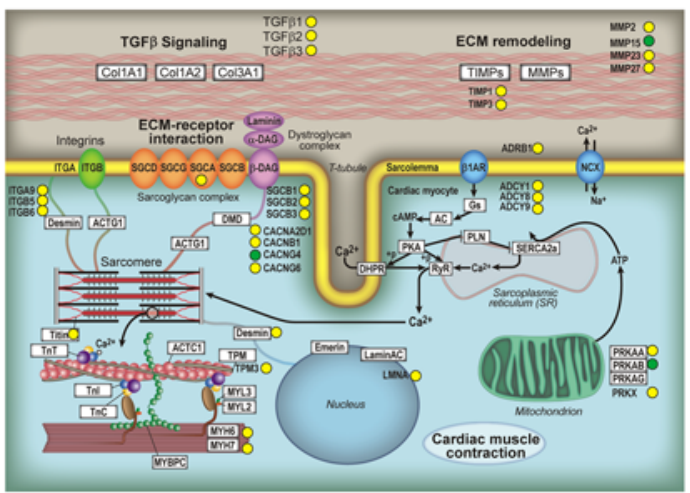

Reversed Incident HF

Persistently Dysregulated

D
C
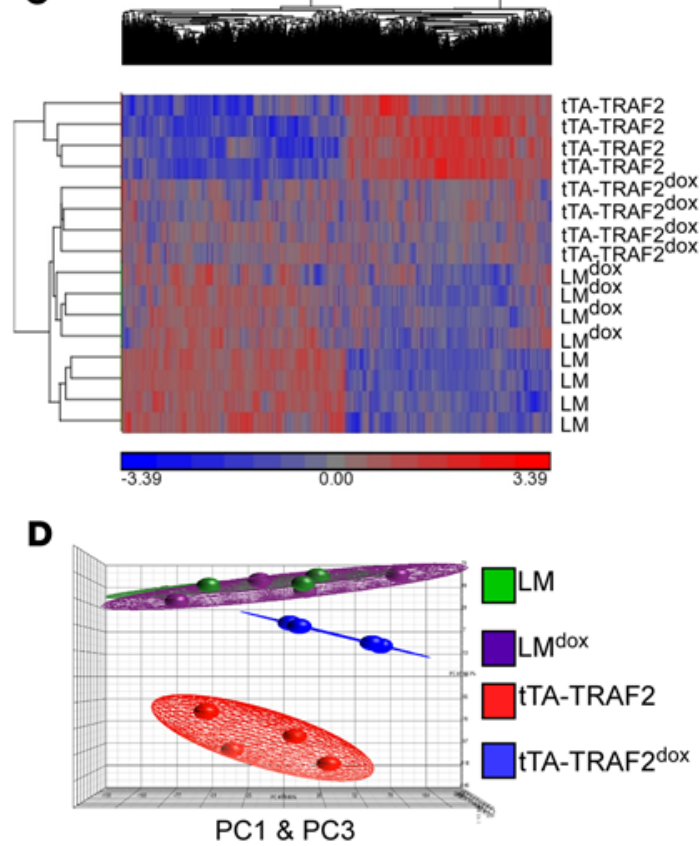

F Matrix/Collagen (40) Integrin/Cytoskeleton (32)

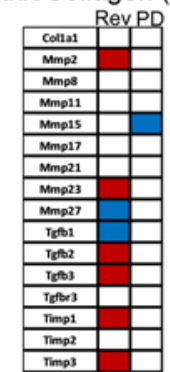

Excitation Contraction/

Coupling (35)

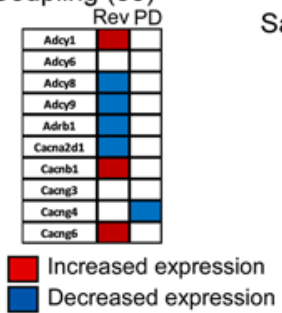

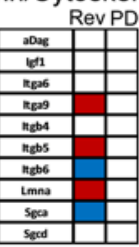

Metabolism/

Energetics (11)
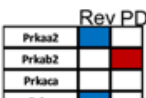

Priba

Sarcomere(15)

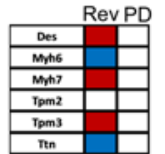
tTA-TRAF2 mice and LM controls were followed up to 8 weeks of age and were then randomized to receive doxycycline in the drinking water or no doxycycline for 8 weeks. (B) Venn diagram of normalized heart failure (HF) genes, persistently dysregulated HF genes, and new reverse remodeled genes (see text for details). (C) Unsupervised hierarchical clustering of changes in gene expression and (D) principal component analysis (PCA) of changes in gene expression. (E) Illustration of persistently dysregulated and reversed HF genes in relation to their anatomic location within the cardiac myocyte. (F) Illustration of persistently dysregulated and reversed HF genes in gene modules relating to cardiac myocyte function (see text for details). The number of genes within each module is given in parenthesis ( $n=4$ hearts per group). Red indicates increased gene expression; blue indicates decreased gene expression relative to LM; Rev, reversed HF genes; PD, persistently dysregulated HF genes. Analysis was performed using Partek Genomic Suite. PC, principal component; PD, persistently dysregulated; Rev, reversed. 
A

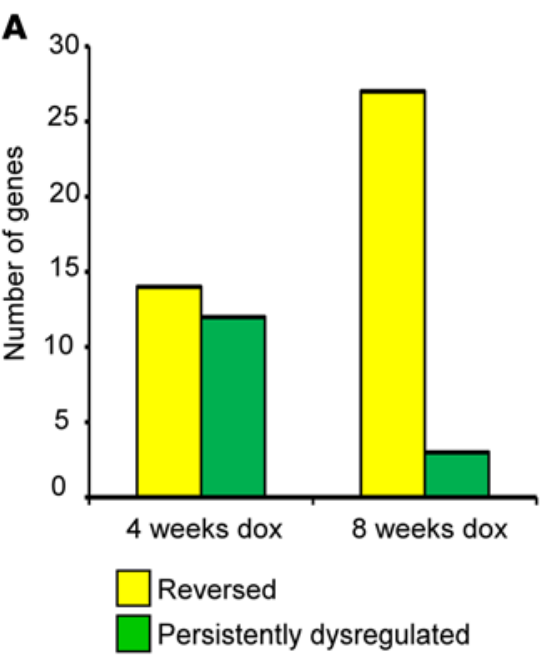

B
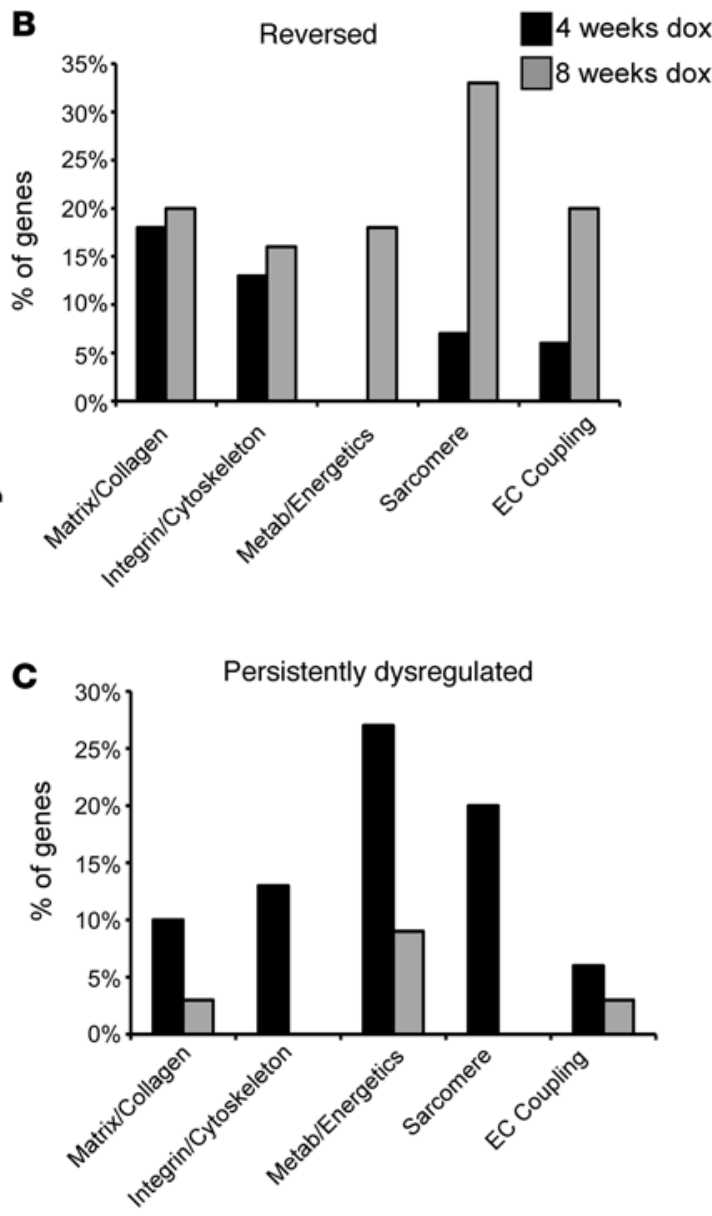

Figure 7. Analysis of reversed and persistently dysregulated genes in 16-week LM, tTA-TRAF2, LM ${ }^{\text {dox8w }}$, and TTA-TRAF2 ${ }^{\mathrm{dox} 8 \mathrm{~W}}$ mouse hearts.

(A) Number of persistently dysregulated and reversed heart failure (HF) genes after 4 and 8 weeks of doxycycline. (B) Percentage of reversed HF genes in functional cardiac myocyte gene modules after 4 and 8 weeks of doxycycline. (C) Percentage of persistently dysregulated HF genes in functional cardiac myocyte gene modules after 4 and 8 weeks of doxycycline ( $n=4$ hearts per group). Analysis was performed using Partek Genomic Suite.

\section{Discussion}

The results of this study - in which we developed and characterized a conditional transgenic mouse model (tTA-TRAF2) that develops reversible DCM - provide 3 unique insights into the biology of reverse LV remodeling. First, normalization of LV structure (Figure 1, E-G) was associated with salutary changes in cardiac myocyte biology (Figure 2, A and B), including reversal of cardiac hypertrophy and improved cardiac myocyte contractile function. The changes in cardiac myocyte biology occurred concurrently with improved LV chamber contractility and reverse LV remodeling (Figure 2, C-E). Importantly, the normalization of LV structure and function was not associated with a single gene, nor a single functional cardiac myocyte gene module. Rather, reversal of the LV HF phenotype was accompanied by a coordinated change in the expression of genes residing within multiple function gene modules (extracellular matrix, integrins/ cytoskeleton, excitation contraction-coupling, and the sarcomere). We also observed the emergence of a number of new genes during reverse LV remodeling, suggesting that reverse LV remodeling is not simply a reversal of the genes that become dysregulated during LV remodeling, but rather is associated with coregulation of distinct sets of novel genes that may be involved in tissue repair. It is noteworthy in this regard that a significant proportion of the new reverse remodeling genes in the tTA-TRAF2 ${ }^{\mathrm{dox} 4 \mathrm{~W}}$ mouse hearts were associated with gene ontologies involved in protein degradation, which are essential for tissue repair (8). To our surprise, reverse LV remodeling in the TRAF2 ${ }^{\mathrm{dox}} 4 \mathrm{~W}$ mouse hearts was not associated with changes in the extracellular matrix (Figure 3), suggesting that improved myocyte contractile function is sufficient to lead to reverse LV remodeling and that changes in fibrillar collagen content are not required. Given that the amount of fibrillar collagen content in the tTA-TRAF2 mice was modest, our findings do not obviate a potentially important role for the reversal of myocardial fibrosis in different experimental models, wherein LV fibrosis is more prominent.

A second important observation was that the complete normalization of LV structure and function in the tTA-TRAF2 $2^{\text {dox }}$ mice was accompanied by a partial $(58 \%)$ normalization of the genes that 
A
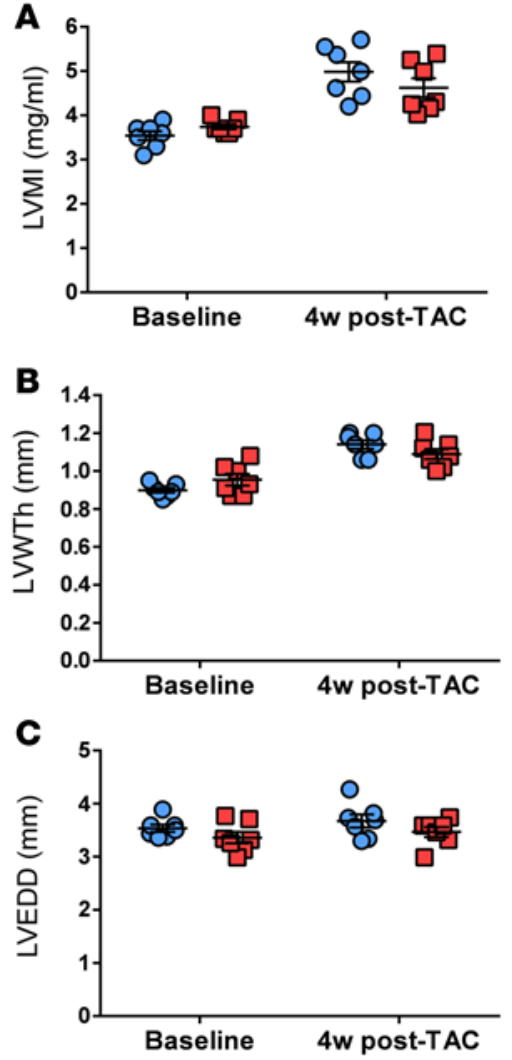

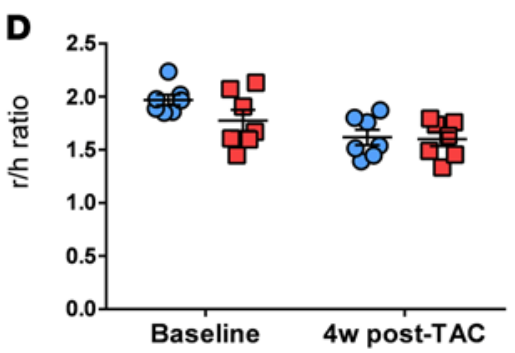

E

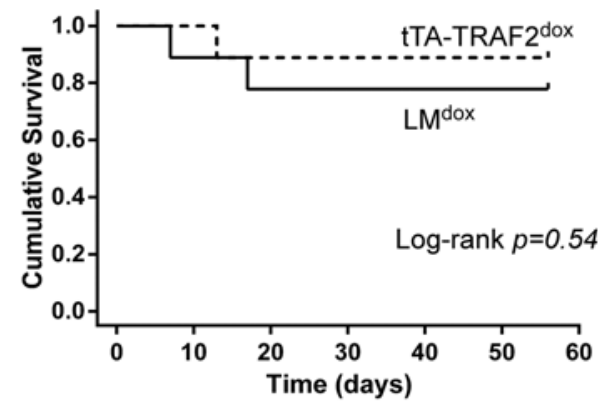

LM $^{\text {dox }}$

TTA-TRAF2 ${ }^{\text {dox }}$

Figure 8. Effect of transaortic constriction (TAC) on LV structure and mortality in LM ${ }^{\mathrm{dox} 8 \mathrm{w}}$ and tTA-TRAF2 ${ }^{\mathrm{dox} 8 \mathrm{w}}$ mice. (A) LV mass index (LVMI), (B) LV wall thickness (LVWTh), (C) LV end diastolic diameter (LVEDD), and (D) LV r/h ratio at 2 and 4 weeks after TAC ( $n=7$ hearts per group). (E) Kaplan Meier analysis of survival in LM ${ }^{\text {dox }}$ and tTA-TRAF2 ${ }^{\text {dox }}$ mice following TAC ( $n=7 \mathrm{LM}^{\mathrm{dox} 8 \mathrm{~W}}$ hearts and $n=7$ tTA-TRAF2 $2^{\mathrm{dox} \times \mathrm{W}}$ hearts). Statistical analysis performed using a repeated measures analysis using mixed models methodology (see Methods section). ( $\left.{ }^{*} P<0.05\right)$.

became dysregulated during LV remodeling. Remarkably, both hierarchical clustering (Figure 4B) and PCA (Figure 4C) revealed that the gene expression profile observed in the tTA-TRAF2 ${ }^{\mathrm{dox} 4 \mathrm{~W}}$ mice more closely resembled the gene profile in the tTA-TRAF2 hearts with an HF phenotype than the control mouse hearts that had normal LV structure and function. Thus, the normalization of LV structure and function is dissociated from reversal of the HF gene program during reverse LV remodeling. Importantly, similar findings with respect to persistent incident HF gene expression were also observed in the failing human hearts from patients that had a DCM whose hearts underwent reverse LV remodeling following LVAD support (Figure 9). Thus, our experimental findings are observed in humans and are likely not model dependent.

The third and perhaps most remarkable finding is that, when the tTA-TRAF2 $2^{\text {dox4w }}$ mice with persistent HF gene expression were stressed by superimposition of a pressure overload, the tTA-TRAF2 ${ }^{\mathrm{dox} 4 \mathrm{~W}}$ mouse hearts developed an exaggerated hypertrophic response at 2 and 4 weeks (Figure 5, A and B) that was associated with increased mortality (Figure 5E). Importantly, the exaggerated hypertrophic phenotype and increased mortality following pressure overload were not observed in the tTA-TRAF2 ${ }^{\mathrm{dox} 8 \mathrm{~W}}$ mice, wherein there was a more complete ( $88 \%$ ) reversal of the incident HF genes and a nearly complete (97\%) reversal of incident HF genes associated with cardiac myocyte contractility, suggesting that a more complete normalization of the HF gene program allows the heart to withstand a second stress imposed by a hemodynamic pressure overload. In this regard, it is interesting to note that cardiac decompensation frequently supervenes in HF patients following LVAD explantation, when the failing heart is exposed to loading conditions that are elevated relative to those encountered during hemodynamic support (9-11).

Reverse $L V$ remodeling. Although reverse LV remodeling has been associated with favorable clinical outcomes in clinical studies, the extant literature suggests that the majority of patients whose hearts 
A Remodeling Reverse Remodeling
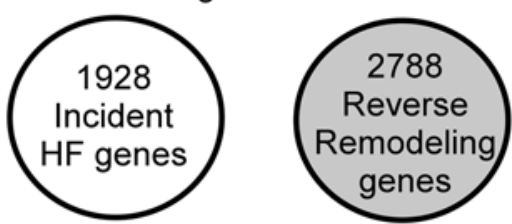

NF vs. DCM

NF vs Post-VAD DCM

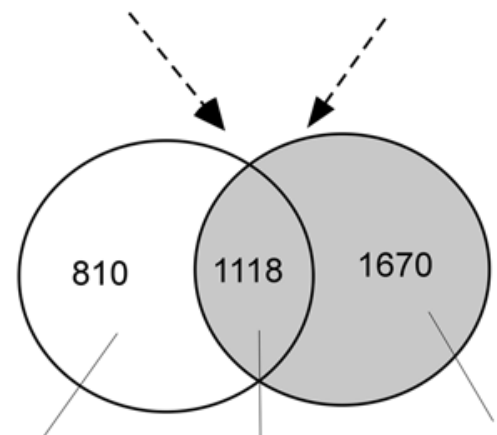

New reverse

Normalized Persistently dysregulated HF genes remodeled genes

B

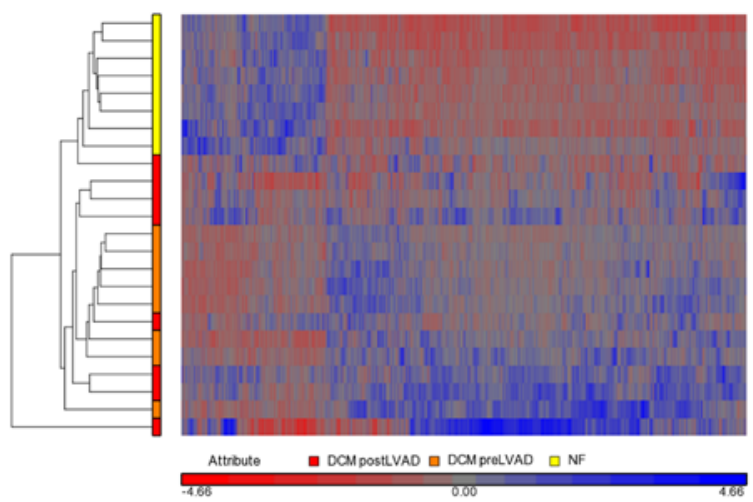

C

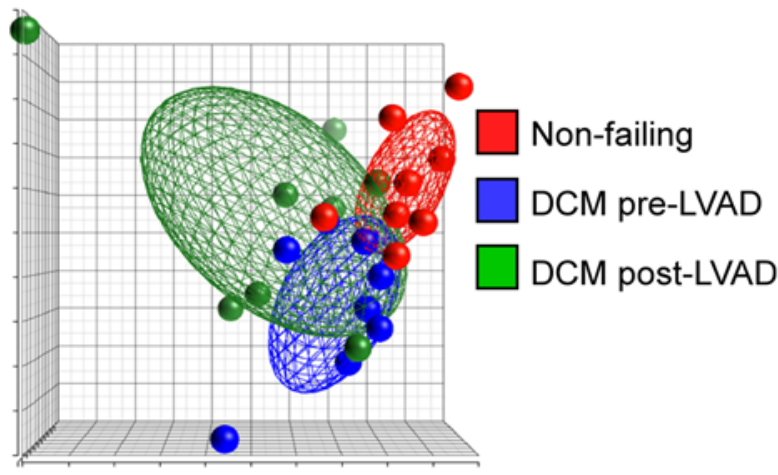

$\mathrm{PC} 1$ \& PC3

E Matrix/Collagen (40) Integrin/Cytoskeleton (32)

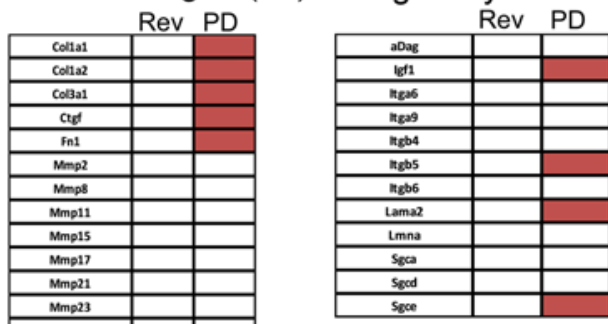

Excitation Contraction/

$\mathbf{F}$
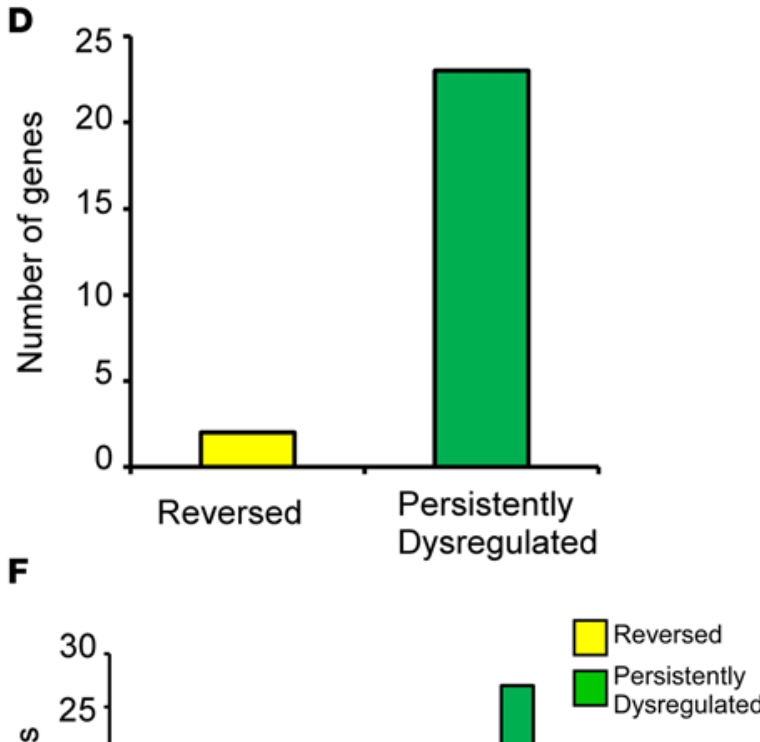
Coupling (35)

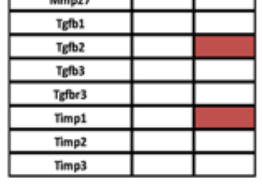

Sarcomere(15)
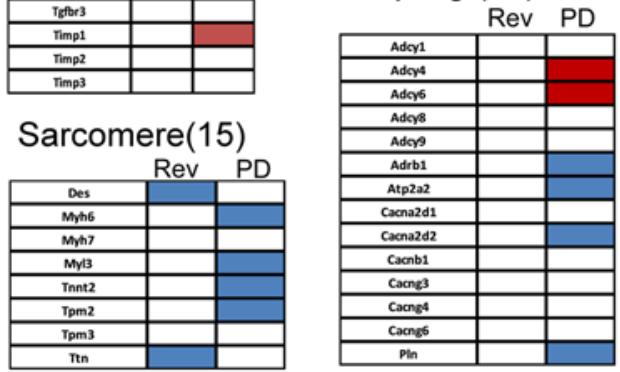

Metabolism/Energetics (11)
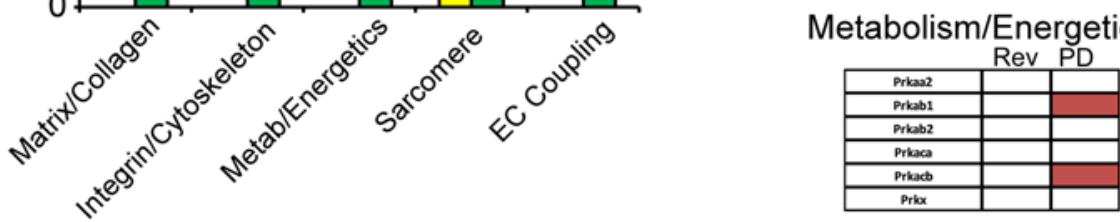
Figure 9. Transcriptional profiling in nonfailing (NF) human hearts $(n=8)$ and paired failing human dilated cardiomyopathy $(D C M)$ hearts $(n=8$ pairs) before and after LVAD. (A) Venn diagram of normalized heart failure (HF) genes, persistently dysregulated HF genes, and new reverse remodeled genes (see text for details). (B) Unsupervised hierarchical clustering of changes in gene expression, and (C) principal component analysis (PCA) of changes in gene expression.(D) Number of persistently dysregulated and reversed HF cardiac myocyte genes. (E) Illustration of persistently dysregulated and reversed HF genes in gene modules relating to cardiac myocyte function (see text for details). The number of genes within each module is given in parenthesis. Red indicates increased gene expression; blue indicates decreased gene expression relative to LM; Rev, reversed HF genes; PD, persistently dysregulated HF genes. Analysis performed using Partek Genomics Suite. (F) Percentage of persistently dysregulated and reversed HF genes in functional cardiac myocyte gene modules after LVAD support. PC, principal component; PD, persistently dysregulated; Rev, reversed.

undergo reverse LV remodeling may suffer from recurrent HF events despite apparent restoration of normal LV structure and function, whereas a smaller number of patients will remain free from recurrent $\mathrm{HF}$ events (3). Although the reasons for these discrepant outcomes are not known, multiple lines of evidence support the point of view that, in most instances, reverse LV remodeling does not lead to a normal heart, despite reversal of many aspects of the HF phenotype. In this regard, one of the more interesting observations has been that the transcriptional changes that occur during reverse LV remodeling are not a simple inverse of the changes that occurred during LV remodeling. Using a variety of different models, including pharmacological (isoproterenol and angiotensin II infusion) (12), transgenic (conditional Akt overexpression) (13), and surgical (thoracic aortic banding and debanding models of reversible cardiac hypertrophy) (14-16), investigators have shown that not only is there a persistence of a subset of genes that become dysregulated during pathological remodeling, but also that there are changes in the expression of genes that are distinct from those that occur during pathological remodeling, indicating that there are unique sets of genes that become altered during the regression of pathological remodeling. Moreover, these genes belong to multiple functional gene ontogenies. Similar observations were reported by Margulies et al., who noted that more than $75 \%$ of the dysregulated genes in failing human HF remained persistently dysregulated or were exacerbated following reversal of the HF phenotype after LVAD support (17); he termed this "molecular hysteresis." Whether this molecular hysteresis represents an adaptive response (i.e., transcriptional plasticity) that allows cardiomyocytes to respond robustly to environmental stress, analogous to the phenomenon of ischemic preconditioning, or whether it represents a maladaptive response because of the inability to return to normalcy is not known.

The results of the present study both confirm and extend prior observations by demonstrating that hearts that have not undergone complete genomic recovery are more vulnerable to a second hemodynamic stress, whereas hearts that have undergone complete transcriptional recovery are able to withstand hemodynamic overloading. The most parsimonious interpretation of these observations is that a more complete reversal of the HF gene program enables the heart to better withstand hemodynamic stress than a partial reversal of the HF gene program, insofar as the persistently dysregulated genes impair cardiac myocyte function and/ or are maladaptive. However, we cannot exclude the formal possibility that some of the persistently dysregulated or new reverse remodeling genes represent "susceptibility genes" that place the heart at risk during hemodynamic overload (e.g., Wnt signaling, MAPK signaling) $(18,19)$. Given that $>55 \%$ of the incident HF genes in the hearts of patients with DCM did not revert to normal following hemodynamic unloading with a LVAD, it is tempting to speculate that one of the reasons these patients cannot be weaned from LVAD support - despite undergoing reverse LV remodeling - is because of the persistence of the abnormal HF gene program. Further studies will be necessary to address this question. Although we did not identify the mechanism(s) for the persistent dysregulated gene expression in the tTA-TRAF2 ${ }^{\mathrm{dox} 4 \mathrm{~W}}$ mouse hearts, one intriguing observation is that changes in the epigenome may contribute to the persistent dysregulated genes.

Conclusion. The results of this study demonstrate that reverse LV remodeling is associated with improvements in the biology of the adult cardiac myocyte, whereas changes in the fibrillar collagen content of the heart are not necessary for restoration of normal LV structure and function. Apart from the importance of these findings, the results of this study also provide a unique glimpse into the biology of the failing heart and show that the failing heart remains vulnerable to hemodynamic stress after restoration of LV structure and function, and that this vulnerability diminishes over time. While our data suggest that this potential window of vulnerability is secondary to an incomplete reversal of the genes that become dysregulated during LV remodeling, we cannot exclude the possibility that abnormalities of the proteome and metabolome also play an important role. Although direct correlations between relatively short-term experimental studies in mice and long-term observations in the humans are not appropriate, these studies do raise the important question of whether recurrent HF hospitalizations, which occur in $\geq 50 \%$ of patients within the first 6 
months of discharge (20), may in some way be related to the susceptibility of the failing myocardium to a subsequent stress in patients who appear clinically stable at the time of discharge from the hospital. Given that recurrent HF hospitalizations are regarded as a measure of poor quality of care, and that health care systems are being penalized for readmitting patients, the current study may have important health care implications in so far as recurrent hospitalization may be the only means to stabilize certain patients, as suggested by several clinical studies $(21,22)$. Finally, these observations may have implications with respect to the development of new therapies that target the ensemble of genes that place HF patients at risk for subsequent hemodynamic deterioration.

\section{Methods}

Generation of tTA-TRAF2 transgenic mice. To develop an experimental model to study reverse LV remodeling, we generated a conditional transgenic mouse model (tTA-TRAF2) that overexpresses TRAF2 in the heart, using a cardiac-specific and tetracycline-transactivating factor-regulated promoter (23). The rationale for conditionally overexpressing TRAF2 in the heart was that we have shown previously that sustained expression of TRAF2, a scaffolding protein that coordinates signaling through the type 1 and type 2 TNF receptors, resulted in LV remodeling and LV dysfunction that phenocopies the LV remodeling and LV dysfunction observed with the development of parainflammation in mice with cardiac restricted overexpression of TNF (4). Further, elevated levels of myocardial TRAF2 have been reported to be elevated in HF patients (24). In this conditional "tet-off" system, the stable tetracycline analog dox (Sigma-Aldrich) inhibits tTA transactivation of the Traf2 transgene in the heart.

Briefly, a murine Traf2 cDNA (4) was cloned into a cardiac-specific, inducibly controlled vector composed of a modified mouse $\alpha$-myosin heavy chain (Myh6) minimal promoter fused with nucleotide binding sites for tTA (23). The transgene construct was injected into single cell embryos of FVB mice at the Baylor College of Medicine (Houston, Texas, USA) transgenic core facility. Founder lines of MYH6-TRAF2 transgenic mice (FVB background) were identified by PCR and confirmed by Southern blotting using a 286 bp probe targeted to exon 8 of the Traf2 gene (Supplemental Figure 4A). Western blotting was performed to confirm the expression of TRAF2 protein in the hearts of 12-week-old MYH6-TRAF2 mouse hearts (Supplemental Figure 4B) using an anti-TRAF2 antibody (sc-877, Santa Cruz Biotechnology Inc.). We obtained 4 founder lines harboring 4, 5, 13, and 29 copies of the Traf 2 transgene. The founder line with 29 copies of the transgene did not yield any progeny; therefore, we selected the MYH6TRAF2 founder line with 13 copies for further characterization. The MYH6-TRAF2 mice were crossed with mice that expressed tTA in the heart (MYH6-tTA [FVB background]), in order to generate lines of tTA-TRAF2 mice that conditionally express TRAF2 in the heart.

In preliminary control experiments, we determined that the cardiac-restricted Traf2 transgene expressed in the tTA-TRAF2 mice was completely silenced 1-2 weeks after adding dox to the drinking water (Supplemental Figure 4B). We further noted that the Traf2 transgene was rapidly reexpressed within 1 week after removing dox from the drinking water (Supplemental Figure 4B). We also characterized lines of LM, MYH6-TRAF2, and MYH6-tTA mice at 8, 12, and 16 weeks of age to determine whether there was a baseline phenotype. As shown in Supplemental Figure 4C, there was no significant difference in HW/BW ratio in the LM, MYH6-TRAF2, and MYH6-tTA mice at any time point. Further, 2-D echocardiography revealed there was no significant difference in the LVEDD nor LV posterior wall thickness in 12-week-old LM, MYH6-TRAF2, and MYH6-tTA mice (Supplemental Figure 4D). As shown in Supplemental Figure 4E, there was no significant difference in LV contractility, measured as LVDP, in 12-week-old LM, MYH6-TRAF2, and MYH6-tTA hearts.

All mice were housed under standard environmental conditions and were fed commercial chow and tap water ad libitum.

Characterization of tTA-TRAF2 transgenic mice. Provisional characterization of the tTA-TRAF2 mice that were fed commercial chow and tap water ad libitum showed that these mice reliably developed a dilated LV HF phenotype by 8 weeks of age. To determine whether this dilated phenotype was reversible, both LM and the tTA-TRAF2 mice were randomized to receive either tap water or tap water with dox $(0.5 \mathrm{mg} / \mathrm{cc}$, changed 3 times weekly) for a period of 4 weeks. Given that the Myh6-tTA transgene has been associated with effects on myocardial gene expression (6), we used MYH6-tTA mice as the appropriate genotype for LM control mice. Four groups of mice were characterized for these studies: LM, LM ${ }^{\text {dox4 }}$, tTA-TRAF2, and tTA-TRAF2 $2^{\text {dox4 }}$. The animals were euthanized at 12 or 16 weeks of age for the purpose of the studies delineated below. 
$L V$ structure. Cardiac hypertrophy was assessed by measuring the HW/BW ratio of the LM, LM ${ }^{\text {dox } 4 \mathrm{~W}}$, tTA-TRAF2, and tTA-TRAF2 ${ }^{\mathrm{dox} 4 \mathrm{w}}$ mice at 12 weeks of age. Two-dimensional-targeted M-mode echocardiography was used to measure LV structure at 8, 12, and 16 weeks of age, as described (25). Anesthesia was induced using $2 \%$ isoflurane and was maintained using $1 \%$ isoflurane. Throughout the 2-D echocardiographic studies, care was taken to maintain the heart rate above $400 \mathrm{bpm}$ by adjusting the level of anesthesia. All 2-D and M-mode echocardiographic measurements were performed by an investigator who was blinded to the experimental group that was being studied.

Isolated cardiac myocyte contractility. Isolated ventricular myocytes were prepared from LM, LM ${ }^{\mathrm{dox} 4 \mathrm{~W}}$, tTA-TRAF2, and tTA-TRAF2 ${ }^{\mathrm{dox} 4 \mathrm{~W}}$ mice at 12 weeks of age, as described (26). Sarcomere length from single cardiac myocytes was recorded using an epi-fluorescence inverted microscope (Nikon TS100) with an attached CCD camera (IonOptix MyoCam, IonOptix). In these experiments, an aliquot of cardiomyocytes was placed into a small superfusion chamber that received a buffer $\left(20 \mathrm{mM} \mathrm{HEPES} \mathrm{[pH} \mathrm{7.4],} 1 \mathrm{mM} \mathrm{CaCl}_{2}\right.$, $137 \mathrm{mM} \mathrm{NaCl}, 5.4 \mathrm{mM} \mathrm{KCl}, 15 \mathrm{mM}$ dextrose, $1.3 \mathrm{mM} \mathrm{MgSO}_{4}$, and $1.2 \mathrm{mM} \mathrm{NaH}_{2} \mathrm{PO}_{4}$ ). All measurements were made on rod-shaped myocytes that had regular well-defined striations. Cardiomyocytes were field stimulated using platinum electrodes. Steady-state trains of stimuli $(2 \mathrm{~ms})$ were applied at a frequency of $0.5 \mathrm{~Hz}$. Sarcomere motion and fluorescence were recorded continuously using IonOptix acquisition software, and the data were stored for an off-line analysis by IonWizard software (IonOptix 1999, version 5). Unloaded sarcomere shortening was calculated based on the difference between peak systolic length and maximum diastolic length.

LV function. Hearts from LM, LM ${ }^{\text {dox4w }}$, tTA-TRAF2, and tTA-TRAF2 ${ }^{\text {dox4 }}{ }^{2}$ mice at 12 weeks of age were freshly excised and buffer perfused in the Langendorff mode, as described (27). The hearts were paced from the right ventricle using a Grass SD9 stimulator (Grass Instruments) at $420 \mathrm{bpm}$. Functional data were recorded at $1 \mathrm{KHz}$ on a data acquisition system (PowerLab, ADInstruments). After 20 minutes of stabilization, the balloon was progressively inflated in $5-\mu 1$ increments until LVDP became asymptotic and then began to decline. LVDP was calculated as the difference between peak-systolic pressure and LVEDP. $\mathrm{LV}$ peak $+\mathrm{LV} \mathrm{dP} / \mathrm{d} t$ and peak $-\mathrm{LV} \mathrm{dP} / \mathrm{d} t$ were determined at peak LVDP.

Myocardial fibrosis. Perfusion-fixed hearts from LM, LM ${ }^{\text {dox4w }}$, tTA-TRAF2, and tTA-TRAF2 ${ }^{\text {dox4w }}$ mice were obtained at 12 weeks and were embedded in paraffin and stained using the picrosirius red technique, as described (28). To calculate the extent of myocardial fibrosis, the LV was divided into 8 quadrants, and the percent area of extracellular picrosirius red staining was computed from random images obtained in each quadrant. Large epicardial arteries and/or veins were excluded, as well as any cutting and/or compression artifacts.

Gene expression profiling. Transcriptional profiling of LM, LM ${ }^{\text {dox } 4}$, tTA-TRAF2, and tTA-TRAF2 ${ }^{\text {dox }}$ mice at 12 and 16 weeks of age was performed using the Sentrix BeadChip and BeadStation system from Illumina Inc. Total RNA was converted to cDNA by reverse transcription using Superscript II reverse transcriptase and T7-(dT)24 primers, followed by second-strand synthesis to generate double-stranded cDNA. After purification, the cDNA was converted to biotin-labeled cRNA, hybridized to a mouse Ref- 8 BeadChip array (Illumina Inc.), and stained with strepavidin-Cy3 for visualization. The mouse Ref- 8 BeadChips contain sequences representing approximately 24,000 curated genes and expressed sequence tags (ESTs). Quality standards for hybridization, labeling, staining, background signal, and basal level of housekeeping gene expression for each chip were verified. After scanning the probe array, the resulting image was analyzed using the GenomeStudio software (Illumina Inc.). Raw expression data was subjected to filtering, log transformation, and quantile normalization for further analysis using PARTEK Genomics Suite (Partek Inc.). Significant genes in LV remodeled hearts (LM vs. tTA-TRAF2) and reverse LV remodeled hearts (LM ${ }^{\text {dox }}$ vs. tTA-TRAF2 ${ }^{\text {dox }}$ ) were identified using ANOVA with contrasts. A fold-change cut-off of 1.2 was utilized in all group comparisons. Agglomerative hierarchical clustering was performed by using an average linkage algorithm with euclidean distance measure based on expression levels of significant HF genes determined by SAM analysis (false called number of 10).

$P C A$. Global mRNA and miRNA expression data from 4 groups of mice were also examined using PCA, which is a mathematical modeling procedure that transforms a number of possibly correlated variables into a smaller number of uncorrelated variables termed principal components (29). PCA allows the complex data sets to be visualized in a simple 3-D display format, wherein the $\mathrm{x}$-axis displays the values for the first principal component, the $y$-axis displays the values for the second principal component, and the $\mathrm{z}$-axis displays the values for the third principal component. Functional annotation clustering of genes differentially expressed dur- 
ing LV remodeling and reverse LV remodeling based on gene ontology classification and the KEGG pathways database was performed using publicly available DAVID v6.7 software (http://david.abcc.ncifcrf.gov/) (30).

To compare the findings obtained in the tTA-TRAF2 mouse hearts with a clinically relevant model of reverse LV remodeling, we examined changes in gene expression in human NF and paired DCM hearts before and after hemodynamic unloading with a LV assist device using deep RNA sequencing, as described (7). Deep sequencing data have been submitted to the National Center for Biotechnology Information gene expression and hybridization array data repository (GEO ID: GSE46224). The duration of LVAD support in this patient cohort was $10.1 \pm 1.7$ months. The myocardial mRNA expression profiles used for the present analysis have been reported previously as an online supplement (see Supplemental Table 6 in ref. 7); however, the informatics analysis of these genes presented here has not been reported previously. Briefly, using 2-tailed adjusted $P<0.05$ and a 1.2-fold change cut-off, we compared changes in gene expression in NF vs. DCM hearts and DCM-LVAD-supported vs. NF LV tissues. Agglomerative hierarchical clustering, PCA, and KEGG pathway analysis were performed as described above.

Transverse aortic constriction. In parallel studies, we performed TAC in $\mathrm{LM}^{\mathrm{dox}}$ and tTA-TRAF2 ${ }^{\mathrm{dox}}$ mice that had received dox in the drinking water from 8-12 weeks of age or from 8-16 weeks of age (31). In brief, mice were anesthetized with a mixture of xylazine $(10 \mathrm{mg} / \mathrm{kg})$ and ketamine $(100 \mathrm{mg} / \mathrm{kg})$, and the TAC procedure was performed by a surgeon blinded to the status of the mice. $\mathrm{LM}^{\text {dox }}$ and tTA-TRAF2 ${ }^{\text {dox }}$ mice subsequently underwent serial 2-D echocardiographic imaging for LV structure and function at 2 and/or 4 weeks after TAC. Echo-imaging for the TAC studies was performed with the mice in a semiconscious state under low dose Avertin anesthesia $(0.05 \mathrm{mg} / \mathrm{g} \mathrm{IP})$. The animals were euthanized 4 weeks following TAC, and the hearts were excised for determination of $\mathrm{HW} / \mathrm{BW}$ ratio.

Statistics. Data are expressed as mean \pm SEM. Two-way ANOVA testing was used to test for timedependent differences in $\mathrm{HW} / \mathrm{BW}$ ratios, 2-D echocardiographic parameters, $\mathrm{LVDP},+\mathrm{dP} / \mathrm{dT},-\mathrm{dP} / \mathrm{dT}$, fibrillar collagen content, and sarcomere shortening between groups; post-hoc comparisons of changes in these parameters between groups at a particular time were performed, where appropriate, using Tukey's test. Analysis of LV structure after TAC was performed using a repeated measures analysis using mixed models methodology. Group, time, and the interaction between group and time were all included in the model. Time (baseline, 1 week, 2 weeks, and 4 weeks) was treated as a categorical variable. Based on the Akaike Information Criteria (AIC) and Shwarz Bayesian Information Critieria (BIC), an autoregressive covariance structure was used to model the repeated measurements. Group means and standard errors were based on model results. Tests to evaluate differences were conducted overall and at each time point. All analysis conducted in SAS v9.4 (SAS Institute Inc.). A $P$ value of $\leq 0.05$ was considered statistically significant in all comparisons.

Study approval. All animal studies conformed with the principles of the NIH Guide for the Care and Use of Laboratory Animals (8th ed. National Academies Press. 2011.) and were approved by the Institutional Animal Care and Use Committee of Baylor College of Medicine and Washington University School of Medicine. All studies using human tissue were conducted in accordance with protocols approved by the Washington University and Columbia University IRBs.

\section{Author contributions}

VKT and KTC were responsible for the majority of the experiments and manuscript generation. KCY performed all of the transcriptional profiling on human samples and performed the analysis of the data. HPT performed the studies on LV mechanics and isolated cell mechanics. SE performed the bioinformatic analysis on the transgenic mice and assisted with manuscript generation. CW and AK performed the surgical procedures and the 2-D echocardiograms and data analysis. JR generated the transgenic tTA transgenic mouse lines and helped with manuscript generation. PB helped with experimental design and manuscript generation. DLM designed the experiments, analyzed the data, and wrote the manuscript.

\section{Acknowledgments}

This research was supported by research funds from the NIH (RO1 HL58081, HL-73017-0, HL089543-01, and T32HL007081).

Address correspondence to: Douglas L. Mann, Cardiovascular Division, 660 South Euclid Ave., Campus PO Box 8066, St. Louis, Missouri 63110-1093, USA. Phone: 314.362.8908; E-mail: dmann@dom.wustl.edu. 
1. Kass DA, et al. Reverse remodeling from cardiomyoplasty in human heart failure. External constraint versus active assist. Circulation. 1995;91(9):2314-2318.

2. Levin HR, Oz MC, Chen JM, Packer M, Rose EA, Burkhoff D. Reversal of chronic ventricular dilation in patients with endstage cardiomyopathy by prolonged mechanical unloading. Circulation. 1995;91(11):2717-2720.

3. Mann DL, Barger PM, Burkhoff D. Myocardial recovery: myth, magic or molecular target? J Amer Coll Cardiol. 2012;60(24):2465-2472.

4. Divakaran VG, et al. Tumor necrosis factor receptor-associated factor 2 signaling provokes adverse cardiac remodeling in the adult mammalian heart. Circ Heart Fail. 2013;6(3):535-543.

5. Panagopoulou $\mathrm{P}$, et al. Desmin mediates TNF- $\alpha$-induced aggregate formation and intercalated disk reorganization in heart failure. J Cell Biol. 2008;181(5):761-775.

6. McCloskey DT, et al. Cardiac transgenesis with the tetracycline transactivator changes myocardial function and gene expression. Physiol Genomics. 2005;22(1):118-126.

7. Yang KC, et al. Deep RNA sequencing reveals dynamic regulation of myocardial noncoding RNAs in failing human heart and remodeling with mechanical circulatory support. Circulation. 2014;129(9):1009-1021.

8. Meier P, Morris O, Broemer M. Ubiquitin-mediated regulation of cell death, inflammation, and defense of homeostasis. Curr Top Dev Biol. 2015;114:209-239.

9. Mancini DM, et al. Low incidence of myocardial recovery after left ventricular assist device implantation in patients with chronic heart failure. Circulation. 1998;98(22):2383-2389.

10. Dandel M, et al. Prediction of cardiac stability after weaning from left ventricular assist devices in patients with idiopathic dilated cardiomyopathy. Circulation. 2008;118(14 supp1):S94-S105.

11. El-Banayosy A, et al. Hemodynamic exercise testing reveals a low incidence of myocardial recovery in LVAD patients. $J$ Heart Lung Transplant. 2001;20(2):209-210.

12. Friddle CJ, Koga T, Rubin EM, Bristow J. Expression profiling reveals distinct sets of genes altered during induction and regression of cardiac hypertrophy. Proc Natl Acad Sci. US A. 2000;97(12):6745-6750.

13. Schiekofer S, Shiojima I, Sato K, Galasso G, Oshima Y, Walsh K. Microarray analysis of Akt1 activation in transgenic mouse hearts reveals transcript expression profiles associated with compensatory hypertrophy and failure. Physiol Genomics. 2006;27(2):156-170

14. Stansfield WE, et al. Regression of pressure-induced left ventricular hypertrophy is characterized by a distinct gene expression profile. J Thorac Cardiovasc Surg. 2009;137(1):232-238.

15. Yang DK, et al. Gene profiling during regression of pressure overload-induced cardiac hypertrophy. Physiol Genomics. 2007;30(1):1-7.

16. Gao XM, et al. Regression of pressure overload-induced left ventricular hypertrophy in mice. Am J Physiol Heart Circ Physiol. 2005;288(6):H2702-H2707.

17. Margulies KB, Matiwala S, Cornejo C, Olsen H, Craven WA, Bednarik D. Mixed messages: transcription patterns in failing and recovering human myocardium. Circ Res. 2005;96(5):592-599.

18. Malekar $\mathrm{P}$, et al. Wnt signaling is critical for maladaptive cardiac hypertrophy and accelerates myocardial remodeling. Hypertension. 2010;55(4):939-945.

19. Flesch M, Margulies KB, Mochmann HC, Engel D, Sivasubramanian N, Mann DL. Differential regulation of mitogen-activated protein kinases in the failing human heart in response to mechanical unloading. Circulation. 2001;104(19):2273-2276.

20. Desai AS, Stevenson LW. Rehospitalization for heart failure: predict or prevent? Circulation. 2012;126(4):501-506.

21. Heidenreich PA, Sahay A, Kapoor JR, Pham MX, Massie B. Divergent trends in survival and readmission following a hospitalization for heart failure in the Veterans Affairs health care system 2002 to 2006. J Am Coll Cardiol. 2010;56(5):362-368.

22. O'Connor CM, Fiuzat M. Is rehospitalization after heart failure admission a marker of poor quality? Time for re-evaluation. $J$ Am Coll Cardiol. 2010;56(5):369-371.

23. Sanbe A, Gulick J, Hanks MC, Liang Q, Osinska H, Robbins J. Reengineering inducible cardiac-specific transgenesis with an attenuated myosin heavy chain promoter. Circ Res. 2003;92(6):609-616.

24. Andrukhova O, et al. Single-dose GSTP1 prevents infarction-induced heart failure. J Card Fail. 2014;20(2):135-145.

25. Baumgarten G, et al. Load-dependent and -independent regulation of proinflammatory cytokine and cytokine receptor gene expression in the adult mammalian heart. Circulation. 2002;105(18):2192-2197.

26. Sakata Y, et al. Toll-like receptor 2 modulates left ventricular function following ischemia-reperfusion injury. Am J Physiol Heart Circ Physiol. 2007;292(1):H503-H509.

27. Knuefermann P, et al. Toll-like receptor 2 mediates Staphylococcus aureus-induced myocardial dysfunction and cytokine production in the heart. Circulation. 2004;110(24):3693-3698.

28. Sivasubramanian $\mathrm{N}$, et al. Left ventricular remodeling in transgenic mice with cardiac restricted overexpression of tumor necrosis factor. Circulation. 2001;104(7):826-831.

29. Ringner M. What is principal component analysis? Nat Biotechnol. 2008;26(3):303-304

30. Dennis G Jr, et al. DAVID: Database for Annotation, Visualization, and Integrated Discovery. Genome Biol. 2003;4(5):P3.

31. DeBosch B, et al. Akt1 is required for physiological cardiac growth. Circulation. 2006;113(17):2097-2104. 